\title{
FOURIER TRANSFORM INFRARED SPECTROSCOPY INVESTIGATION OF \\ WATER MICROENVIRONMENTS IN POLYELECTROLYTE MULTILAYERS AT \\ VARYING TEMPERATURES
}

\author{
A Thesis \\ by \\ CHIKAODINAKA IFUNANYA ENEH
}

\begin{abstract}
Submitted to the Office of Graduate and Professional Studies of Texas A\&M University in partial fulfillment of the requirements for the degree of

MASTER OF SCIENCE
\end{abstract}

Chair of Committee, Jodie L. Lutkenhaus

Committee Members, Yossef A. Elabd Svetlana Sukhishvili

Head of Department, Arul Jayaraman

August 2020

Major Subject: Chemical Engineering Copyright 2020 Chikaodinaka Ifunanya Eneh 


\section{ABSTRACT}

Polyelectrolyte multilayers (PEMs) are thin films formed by the alternating deposition of positively and negatively charged polyelectrolytes. They have become materials of great interest over the past few decades due to their highly adaptive properties. Water plays an important role in influencing the physical properties of PEMs, as it can act both as a plasticizer and swelling agent. However, the way in which water molecules distribute around and hydrate ion pairs has not been fully studied. In this thesis, the influence of ionic strength and temperature on PEM water microenvironments is studied. Here, we examine the effects of temperature and ionic strength on the hydration microenvironments of fully immersed poly(diallyldimethylammonium)/ polystyrene sulfonate (PDADMA/PSS) PEMs. This is accomplished by tracking the OD stretch peak using attenuated total reflectance Fourier transform infrared (ATR-FTIR) spectroscopy at $0.25 \mathrm{M}-1.5 \mathrm{M} \mathrm{NaCl}$ and $35-70{ }^{\circ} \mathrm{C}$. The OD stretch peak is deconvoluted into three peaks: 1) high frequency water represents tightly bound water, 2) low frequency water represents loosely bound water, and 3) bulk water represents water with little or no binding to ion pairs. In general, the majority of water absorbed into the PEM exists in a bound state, with little-to-no bulk water observed. As temperature increases, PDADMA/PSS PEM loses a small amount of water; meanwhile, the high frequency water maintains its peak area while the peak center shifts to the right. This indicates that tightly bound water becomes even more tightly bound with no change in amount. The effect of ionic strength on the PDADMA/PSS PEM is more nuanced and follows a trend influenced by competing 
effects of electrostatic screening and repulsion. A van't Hoff plot obtained from the ratio of the amount of high frequency water to low frequency water at each temperature shows that the amount of energy required to exchange between water states is from $11-22 \mathrm{~kJ} / \mathrm{mol}$ across the studied range of salt concentration. The entropy gain associated with this transition is $48-79 \mathrm{~kJ} / \mathrm{molK}$. These results provide quantitative information on the association of water molecules within PEMs and serves as a precursor to understanding these materials' ion-water microenvironments with respect to temperature and ionic strength. 


\section{DEDICATION}

This thesis is dedicated to my mother, Regina Eneh

and my sisters, Nneka Agujiobi and Uju Chime

for your constant emotional, moral, spiritual and financial support.

I couldn't have asked for a better family. 


\section{ACKNOWLEDGEMENTS}

I would like to show deep gratitude to Dr Jodie L. Lutkenhaus for taking me under her mentorship and accepting me into her research group. She has been supportive, patient, and inspiring. She availed herself to multiple one-on-one sessions to provide guidance in the early stages of my program and trusted in my abilities to grow into an independent researcher. Her mentorship has provided me with all-round growth in research, technical communication and leadership.

I would like to thank Dr. Yossef Elabd and, Dr. Svetlana Sukhishvili and Dr. Zhengdong Cheng for taking time to serve on my committee.

I also would like to thank our collaborators, Dr. Maria Sammalkorpi and Dr Piotr Batys for their contributions to this research project. They constantly provide a valuable insight for better understanding of our experimental results through computations.

I would also like to express my appreciation to my lab members, both old and new, for giving me a family at work. Special thanks to Dr Pilar Suarez-Martinez for her careful mentorship, taking time to show me step-by-step procedures in the lab and out of the lab. I am so fortunate to work with many great people and make friends with them. They have helped me surpass hurdles research presents daily.

Finally, I want to thank my family and friends for their always support. I would like to thank my mother. She ensured she remained close to me at heart even though miles away. My family have provided all I needed to pursue this dream with strength and confidence. I would like to thank my friends from undergraduate for their understanding, love and support. Their virtual presence gave me something familiar to hold on to when 
everything was changing. I would love to thank the friends I have made here at Texas A\&M University for making graduate school a fun experience amidst its burdens.

Most importantly, I would love thank God for my existence and this amazing opportunity to invest my share of His talents given to me. 


\section{CONTRIBUTORS AND FUNDING SOURCES}

\section{Contributors}

This work was supported and supervised by a thesis committee consisting of Professors Jodie Lutkenhaus, Yossef Elabd of the Department of Chemical Engineering and Professor and Svetlana Sukhisvili of the Department of Materials Science and Engineering. FTIR experiments were carried out by Matthew Bolen and all other analysis and work was carried out by student independently.

\section{Funding Sources}

Graduate study and this work were supported by the National Science Foundation under Grant Number 1609696. 


\section{NOMENCLATURE}

\begin{tabular}{ll} 
A & Absorbance \\
ATR-FTIR & Attenuated total reflectance- Fourier transform infrared \\
BPEI & Branched polyethyleneimine \\
c & Speed of light \\
c & concentration of the sample \\
$\mathrm{CO}_{2}$ & Carbon dioxide \\
$\mathrm{D}_{2} \mathrm{O}$ & Deuterium oxide \\
$\mathrm{E}$ & Energy \\
$\mathrm{f}$ & Frequency \\
$\mathrm{FTIR}$ & Fourier transform infrared \\
$\mathrm{FWHM}$ & Full width half max \\
$\mathrm{h}$ & Planck's constant \\
$\mathrm{HOD}$ & Prepared by mixing $5 \%$ v/v D2O to H2O \\
$\mathrm{I}$ & Intensity of transmitted beam \\
$\mathrm{I}_{\mathrm{O}}$ & Intensity of incident beam \\
$\mathrm{IR}$ & Infrared \\
$\mathrm{KBr}$ & Potassium bromide \\
$\mathrm{l}$ & Path length \\
$\mathrm{LbL}$ & Layer by layer \\
$\mathrm{MDSC}$ & Modulated differential scanning calorimetry \\
& \\
\hline &
\end{tabular}




\begin{tabular}{ll} 
MMT & Montomorillonite \\
NaCl & Sodium chloride \\
NMR & Nuclear magnetic resonance \\
NR & Neutron reflectometry \\
PAA & Poly(acrylic acid) \\
PAH & Poly(allylamine hydrochloride) \\
PDADMA & Poly(diallyldimethyl ammonium chloride) \\
PE & Polyelectrolyte \\
PEC & Polyelectrolyte complex \\
PEM & Polyelectrolyte multilayer \\
PMAA & Poly(methacrylic acid) \\
PSS & Polystyrene sulfonate \\
QCM & Quartz crystal microbalance \\
QPVP & Quaternized poly(4-vinylpyridine) \\
RH & Relative humidity \\
T & Transmittance \\
v & Wavenumber \\
XRR & X-ray reflectometry \\
ZnSe & Zinc selenide \\
$\varepsilon$ & Molar absorptivity \\
$\lambda$ & Wavelength \\
\hline
\end{tabular}




\section{TABLE OF CONTENTS}

Page

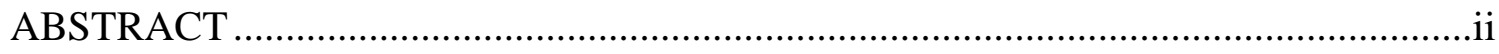

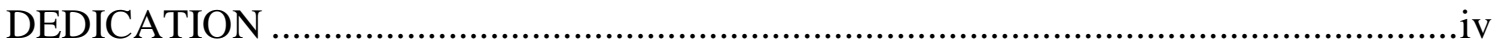

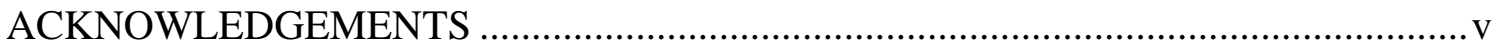

CONTRIBUTORS AND FUNDING SOURCES ...................................................vii

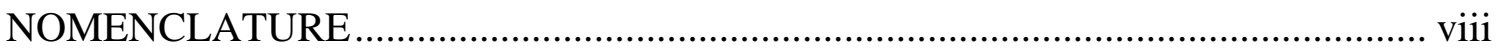

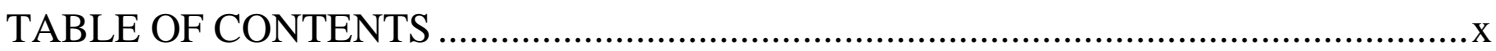

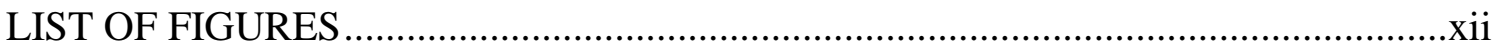

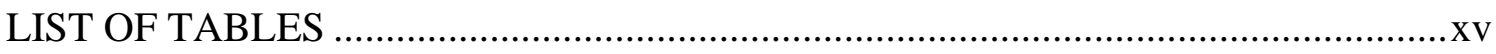

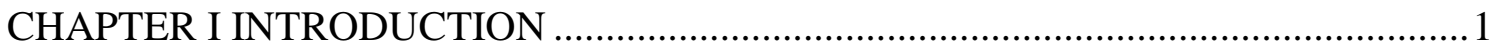

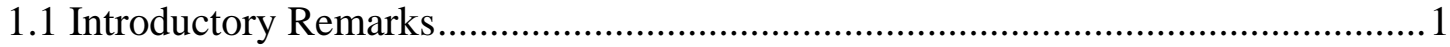

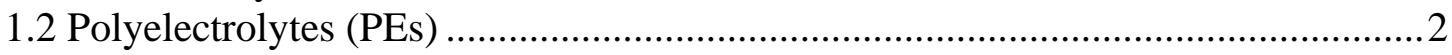

1.2.1 Natural and Synthetic polyelectrolytes.................................................... 3

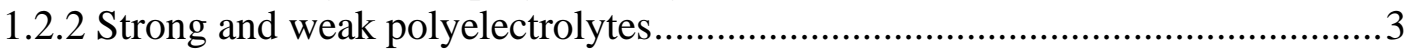

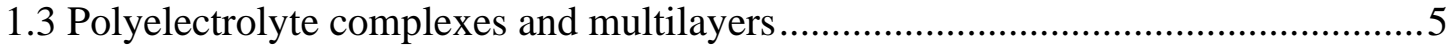

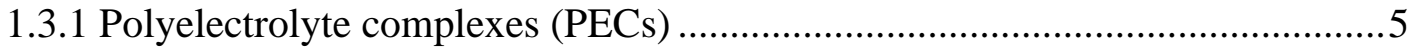

1.3.2 Polyelectrolyte multilayers (PEMs) .................................................... 7

1.3.3 Comparing PECs and PEMs.................................................................

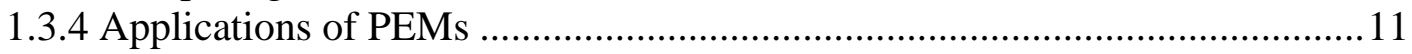

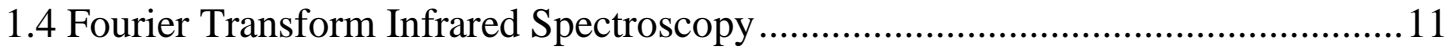

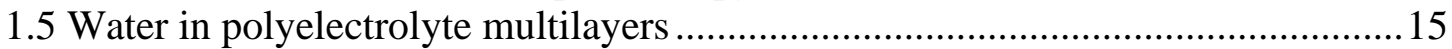

1.5.1 Water content of polyelectrolyte multilayers .......................................... 15

1.5.2 Water micro-environments in PEMs ....................................................... 17

1.5.3 Effects of ionic strength on water content of PEMs.....................................22

1.5.4 Effects of temperature on water content of PEMs .....................................24

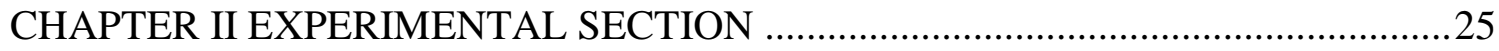

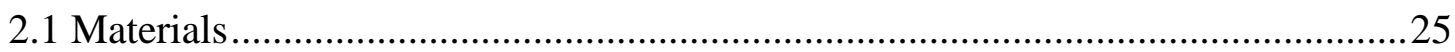

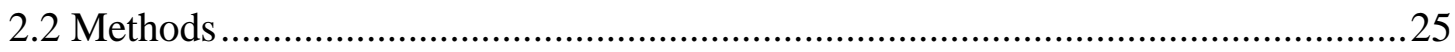




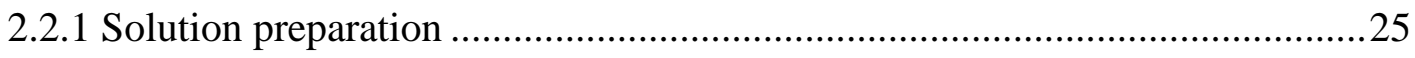

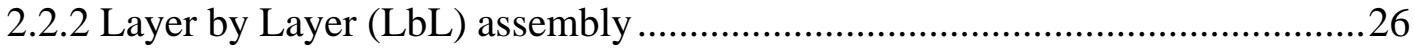

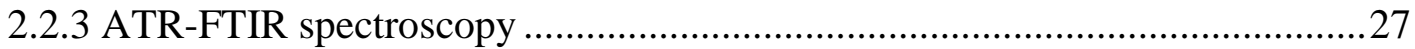

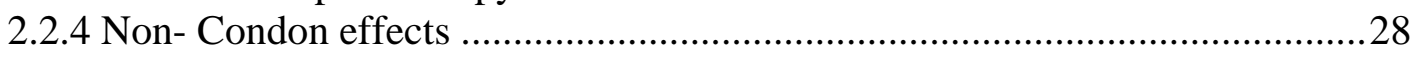

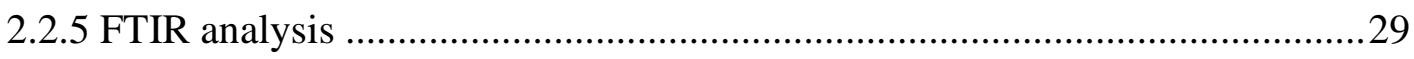

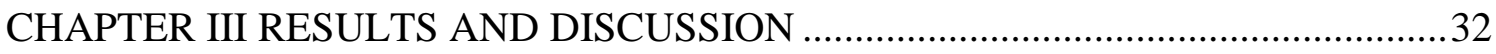

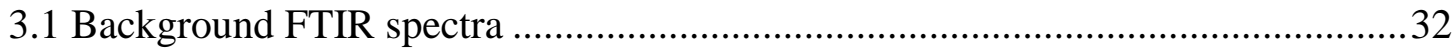

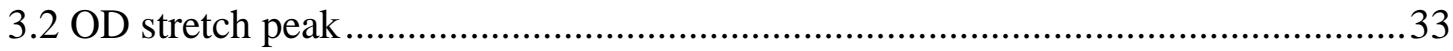

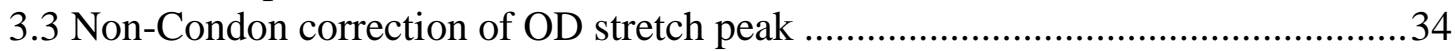

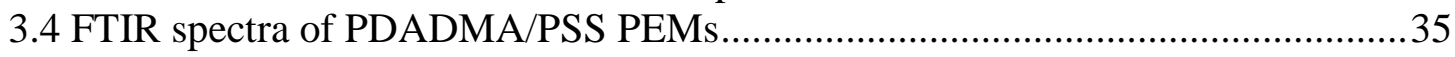

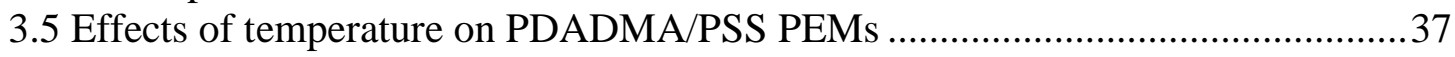

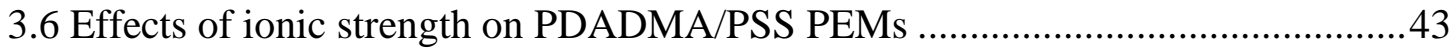

3.7 Combined effects of temperature and ionic strength on PEMs ............................49

CHAPTER IV CONCLUSIONS AND FUTURE WORK ............................................54

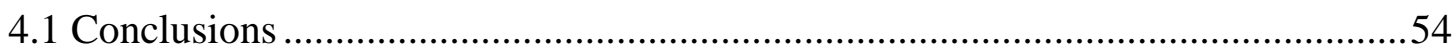

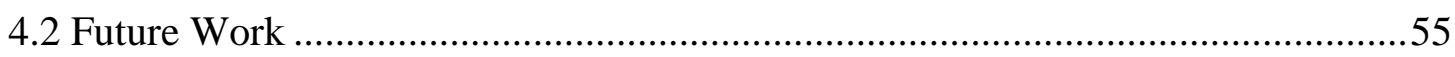

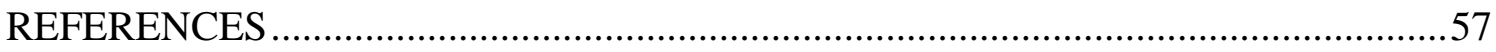




\section{LIST OF FIGURES}

Page

Figure 1.1: Examples of strong and weak polyelectrolytes

Figure 1.2: Polyelectrolyte complex preparation .5

Figure 1.3: Schematic representation of the processes used to fabricate polyelectrolyte multilayer films by LbL assembly. (a) Dipping LbL assembly. (b) Spinassisted LbL assembly. (c) Spray-assisted LbL assembly. Multilayer films are formed by repeating steps 1 to 4 in a cycle fashion. Reproduced from Ref. [40] "Layer-by-layer assembly for rapid fabrication of thick polymeric films" by Li, Y., Wang, X., \& Sun, J., 2012. Chemical Society Reviews, 41(18), 5998-6009 with permission from The Royal Society of Chemistry.

Figure 1.4: Comparison of polyelectrolyte complexes and multilayers. 9

Figure 1.5: Electromagnetic spectrum. Figure reproduced with permission from ref $\left[{ }^{51}\right]$ "Biological effects of non-ionizing electromagnetic fields: Two sides of a coin" by Saliev, T., Begimbetova, D., Masoud, A.-R., \& Matkarimov, B., 2019. Progress in Biophysics and Molecular Biology, 141, 25-36 by Elseveir,

Figure 1.6: Working principle of ATR-FTIR

Figure 1.7: Swelling and void water in PEMs. Reprinted from reference [74] "About different types of water in swollen polyelectrolyte multilayers" by Koehler, R., Steitz, R., \& von Klitzing, R., 2014. Advances in Colloid and Interface Science, 207, 325-331 with permission from Elsevier.

Figure 1.8: Freezing free, freezing bound and non-freezing bound water. Reprinted with permission from [75] "Hydration and Temperature Response of Water Mobility in Poly(diallyldimethylammonium)-Poly(sodium 4styrenesulfonate) Complexes" by Batys, P., Zhang, Y., Lutkenhaus, J. L., \& Sammalkorpi, M., 2018. Macromolecules, 51(20), 8268-8277. Copyright (2018) American Chemical Society https://pubs.acs.org/doi/full/10.1021/acs.macromol.8b01441 ........................22

Figure 2.1: Schematic of layer by layer assembly of PDADMA/PSS films..................27

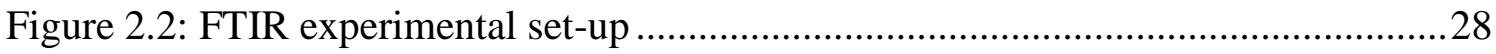

Figure 2.3: (a) A sample spectrum showing deconvolution of the OD asymmetrical stretching region from 2400 to $2700 \mathrm{~cm}^{-1}$ of PDADMA/PSS PEM immersed 
in $0.50 \mathrm{M} \mathrm{NaCl} \mathrm{HOD} \mathrm{solution.} \mathrm{The} \mathrm{three} \mathrm{peaks} \mathrm{(in} \mathrm{blue,} \mathrm{green,} \mathrm{and} \mathrm{red)}$ correspond to populations of HOD in different states of association within the surrounding environment: bulk water, low frequency water, and high frequency water. (b) A visual representation of the water microenvironments and their distribution around both intrinsic and extrinsic ion pairs showing low frequency and high frequency water.

Figure 3.1: FTIR spectra of bare ZnSe crystal at different temperatures $\left(35-70{ }^{\circ} \mathrm{C}\right.$ at $5{ }^{\circ} \mathrm{C}$ intervals).

Figure 3.2: Comparison of $5 \mathrm{wt} \% \mathrm{D}_{2} \mathrm{O}$ in $\mathrm{H}_{2} \mathrm{O}$ showing lack of interfering peaks in the OD region $\left(2700-2400 \mathrm{~cm}^{-1}\right)$. Spectra were not corrected for non-Condon effects

Figure 3.3: Shape comparison disregarding peak height shows the shift of the overall peak maximum towards higher frequencies when spectra are corrected for non-Condon effects as described in the materials and methods section. Uncorrected left $\mathrm{Y}$-axis, Non-Condon corrected right $\mathrm{Y}$-axis. Data corresponds to $0.25 \mathrm{M} @ 35^{\circ} \mathrm{C}$.

Figure 3.4: Set of baselined FTIR spectra $\left(400-4000 \mathrm{~cm}^{-1}\right)$ for a 70-layer pair PDADMA/PSS film assembled in $0.50 \mathrm{M} \mathrm{NaCl}$ solution and immersed in $0.50 \mathrm{M} \mathrm{NaCl} \mathrm{HOD}$ solution at temperatures from $35-65^{\circ} \mathrm{C}$.

Figure 3.5: Set of baselined FTIR spectra of the polymer fingerprint region (1000 $1500 \mathrm{~cm}^{-1}$ ) of a 70-layer pair PDADMA/PSS film assembled in $0.50 \mathrm{M} \mathrm{NaCl}$ solution and immersed in $0.50 \mathrm{M} \mathrm{NaCl} \mathrm{HOD} \mathrm{solution} \mathrm{at} \mathrm{temperatures} \mathrm{from}$ $35-65^{\circ} \mathrm{C}$

Figure 3.6: Set of baselined FTIR spectra of a) the OH stretching region (2800 - 3800 $\left.\mathrm{cm}^{-1}\right)$, b) the OD stretching region $\left(2400-2700 \mathrm{~cm}^{-1}\right.$ ) of a 70-layer pair PDADMA/PSS film assembled in $0.50 \mathrm{M} \mathrm{NaCl}$ solution and immersed in $0.50 \mathrm{M} \mathrm{NaCl} \mathrm{HOD}$ solution at temperatures from $35-65^{\circ} \mathrm{C}$

Figure 3.7: Combined deconvolutions for a PEM immersed in HOD solutions of different salt concentrations with arrows indicating the change in peak position as temperature increases from $35^{\circ} \mathrm{C}$ to $70{ }^{\circ} \mathrm{C}$. a) $0.25 \mathrm{M}$, b) $0.50 \mathrm{M}$, c) $0.75 \mathrm{M}$, d) $1.00 \mathrm{M}$, e) $1.25 \mathrm{M}$, and f) $1.50 \mathrm{M}$. Legend in (f) applies to all panels.

Figure 3.8: FTIR spectra of fingerprint region at varying salt concentrations.

Figure 3.9: A relationship between the $\mathrm{SO}_{3}{ }^{-}$anti-symmetric stretch peak (at $1191 \mathrm{~cm}^{-}$

${ }^{1}$ ) and salt concentration obtained from Figure 3.8. 
Figure 3.10: a) Combined deconvolutions for a PEM immersed in HOD solutions of different salt concentrations at $40{ }^{\circ} \mathrm{C}$ showing the cumulative (black), high (red) and low frequency water (green), and bulk water (blue) peaks. b) Cumulative c) Low frequency d) High frequency water peaks at different salt concentrations at $40^{\circ} \mathrm{C}$ obtained from Figure 3.10a for clarity. The arrows in (b) and (d) indicate the trend with increasing salt concentration.

Figure 3.11: (a) Population distribution of water as percent area of each state for all tested salt concentrations and temperatures. (b) Comparison of percent area of low and high frequency peaks with respect to increasing salt concentration at $40^{\circ} \mathrm{C}$ (black) and $60^{\circ} \mathrm{C}$ (red). The two top-most (higher percentage) lines correspond to the high frequency water peaks and the two bottom-most (lower percentage) correspond to the low frequency water peaks.

Figure 3.12: van't Hoff plot produced using the percent areas from Figure 3.11. The lines represent linear fits using the van't Hoff relationship. b) van't Hoff enthalpy dependence on salt concentration 


\section{LIST OF TABLES}

Page

Table 3.1: Peak information for all temperatures at $0.25 \mathrm{M} \mathrm{NaCl}$...............................41

Table 3.2: Peak information for all salt concentrations at $40{ }^{\circ} \mathrm{C}$.................................48 


\section{CHAPTER I}

\section{INTRODUCTION}

\subsection{Introductory Remarks}

Polyelectrolyte complexes and multilayers (PECs and PEMs) are formed by the simultaneous electrostatic attraction between polymers of opposite charge and the entropic expulsion of counterions. The earliest report of polyelectrolyte complexation was by Bungenberg de Jong et al. in 1929, where he observed a phase separation upon mixing a polycation with a polyanion. ${ }^{1}$ In general, complexation may lead to a solid-liquid or liquidliquid phase separation depending on the conditions of preparation. As a result, PECs are highly tunable with properties most commonly controlled by $\mathrm{pH}$, salt, water and temperature.

By virtue of their behavioral flexibility, PECs and PEMs have been studied for applications in a wide range of fields. From potential applications involving their use as separators in batteries ${ }^{2}$ to protective coatings ${ }^{3-5}$ to stimuli-responsive drug delivery and bio-compatible coatings ${ }^{6,7}$, PECs and PEMs are worth the amount of attention they've received over the past years.

PECs are formed by simultaneous mixing of both polycation and polyanion whereas PEMs are prepared by an alternating layer by' layer (LbL) assembly. Both preparation techniques occur in aqueous media, thereby incorporating water as a one of the major components in both PECs and PEMs. 
In this thesis, we study the influence of temperature and ionic strength on water uptake in PEMs. We then take a step further to group water into three possible water microenvironments within PEMs and equally study the influences both temperature and ionic strength.

\subsection{Polyelectrolytes (PEs)}

Polyelectrolytes are polymers which possess ionizable functional groups in their repeat structure. ${ }^{8}$ Just as typical electrolyte can be acids, bases and salts, polyelectrolytes can also be polycations, polyanions or polysalts. However, a polyelectrolyte differentiates from a simple electrolyte in that it is a macromolecule comprised of many repeat units. Also, polyelectrolytes dissociate when dissolved in water to form polymer that bears a charge while releasing counterions and this differentiates them from neutral polymers. ${ }^{9}, 10$

The sulfonate $\left(-\mathrm{SO}_{3}{ }^{-}\right)$, carboxylate $\left(-\mathrm{COO}^{-}\right)$, and phosphonate $\left(-\mathrm{PO}_{3} \mathrm{H}^{-},-\mathrm{PO}_{3}{ }^{2-}\right)$ are by far some of the most ubiquitous anionic functional groups while for cationic groups primary, secondary and quaternary ammonium $\left(-\mathrm{NH}_{3}{ }^{+}, \quad=\mathrm{NH}_{2}{ }^{+} \& \quad \equiv \mathrm{N}^{+}\right)$for common. ${ }^{11}$ Often times these functional groups are found as a side group to the main polyelectrolyte backbone but can be found within the polyelectrolyte backbone. In this case, the polyelectrolytes are called polyionenes. ${ }^{11}$

The most important property of polyelectrolytes is its solubility in water governed by its hydrophobicity or hydrophilicity. ${ }^{9}$ While most natural polyelectrolytes are soluble in water, many synthetic PEs are not because of the repulsions between hydrophobic backbone and water. 9,12 This challenge can be resolved by adding more ionic components 
to polyelectrolyte charge, that is increasing the charge density. ${ }^{9,}{ }^{13}$ Changes in degree of dissociation have an influence on polymer chain size, end-end distance, radius of gyration and overall chain conformation. ${ }^{14}$

All polyelectrolytes, including both polycations and polyanions can be classified into groups on a couple of bases. The most common are source and charge density of polyelectrolyte.

\subsubsection{Natural and Synthetic polyelectrolytes}

One way of classifying polyelectrolytes would be broadly into 2 groups of natural and synthetic polyelectrolytes. Natural polyelectrolytes such as polypeptides, DNA, chitosan (polycation) and sodium alginate (polyanion) can be extracted from both plant and animal sources. ${ }^{12}$ They are usually considered bio-compatible with human body from drug delivery systems and bio-coatings. ${ }^{12}$ Synthetic polyelectrolytes, on the other hand, are produced as a result of polymerization reactions. ${ }^{15}$ Of the synthetic polyelectrolytes, the most commonly studied are poly(diallyldimethyl ammonium chloride) (PDADMA), poly(styrene sulfonate) (PSS), poly(allyamine hydrochloride) (PAH), poly(acrylic acid) (PAA). ${ }^{16-21}$

\subsubsection{Strong and weak polyelectrolytes}

When polyelectrolytes dissolve in water, they dissociate into their charged states; another method of polyelectrolyte classification is based on the degree of this dissociation in water. Strong polyelectrolytes completely dissociate in water and their charge densities 
are independent of $\mathrm{pH} .{ }^{15}$ The dissociation constant, $\mathrm{pKa}$, of weak polyelectrolytes, on the other hand, can be controlled by $\mathrm{pH} .^{22}$

On their own, polyelectrolytes have been applied as excellent flocculants or dispersant in water purification, oil recovery and mineral processing. ${ }^{15}$ Figure 1.1 shows 4 examples of synthetic polyelectrolytes classified by their ability to dissociate in water. In this thesis, we work with strong synthetic polyelectrolytes, PDADMA and PSS.

\section{Polycation}

\section{Strong polyelectrolyte}

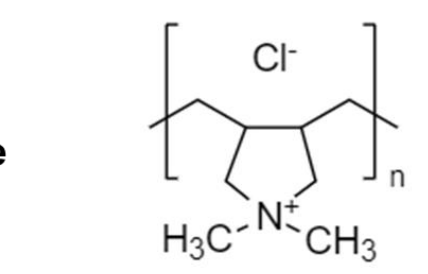

Poly(diallyldimethyl ammonium chloride) (PDADMA)

\section{Weak polyelectrolyte}

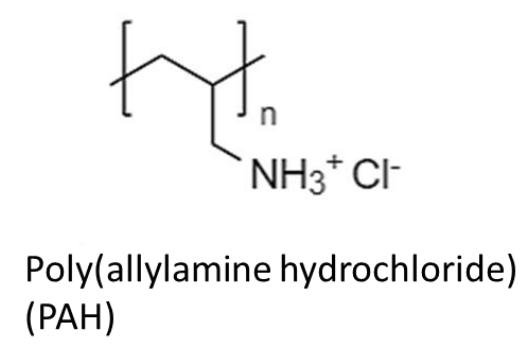

\section{Polyanion}

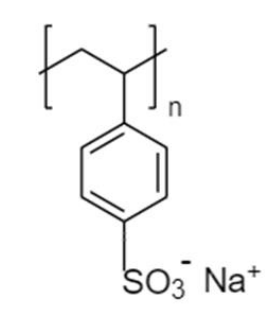

Poly(sodium 4-styrene sulfonate) (PSS)

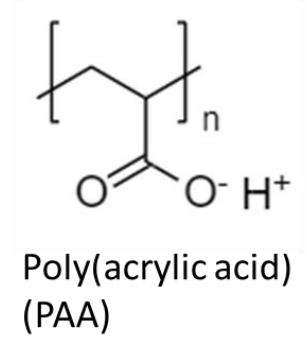

Figure 1.1: Examples of strong and weak polyelectrolytes 


\subsection{Polyelectrolyte complexes and multilayers}

\subsubsection{Polyelectrolyte complexes (PECs)}

Polyelectrolyte complexes (PECs) are formed by the simultaneous mixing of oppositely charged polyelectrolytes causing an entropic expulsion of small counterions as shown in Figure 1.2. This interaction is described by equation 1.1. The PECs then remain bonded by electrostatic forces, in addition to weaker hydrogen bonds and hydrophobic forces exist within PEMs.

$$
\mathrm{Pol}^{+} A^{-}{ }_{a q}+\mathrm{Pol}^{-} \mathrm{M}^{+}{ }_{a q} \rightarrow \mathrm{Pol}^{+} \mathrm{Pol}^{-}{ }_{s}+A^{-}{ }_{a q}+\mathrm{M}^{+}{ }_{a q}
$$

Where $\mathrm{Pol}^{+}$and $\mathrm{Pol}^{-}$represent polycation and polyanion And $\mathrm{A}^{-}$and $\mathrm{M}^{+}$represent associated counterions

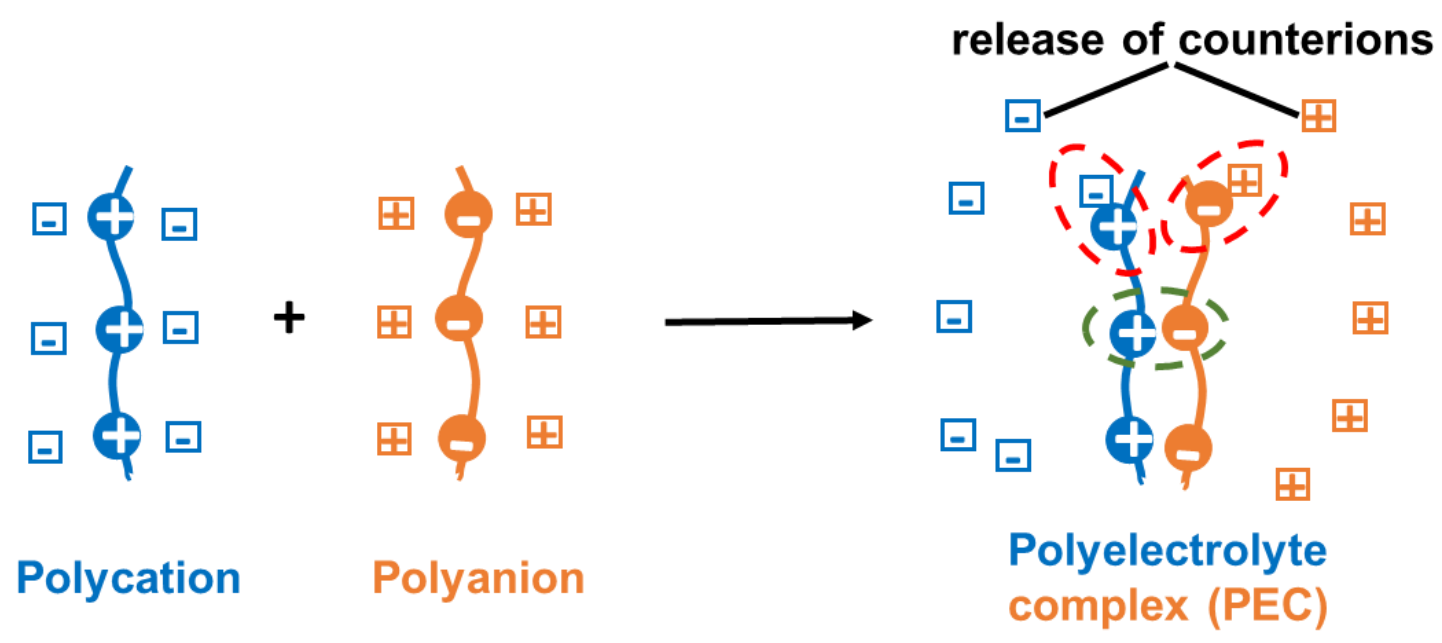

Figure 1.2: Polyelectrolyte complex preparation 
In the presence of salt ions, polyelectrolyte ion pairing is hindered by the breaking of polyelectrolyte-polyelectrolyte (intrinsic) ion pairs to form polyelectrolyte-salt (extrinsic) ion pairs. The green circles with broken lines surround an intrinsic ion pair whereas the red circles surround extrinsic ion pairs in the Figure 1.2. This can be described by the reverse of equation 1.1 expressed as equation 1.2 below. As a result of extrinsic ion pair formation, PECs exhibit 3 phases with increasing salt content; namely a solid precipitate phase, liquid-liquid phase separated coacervate phase, and a solution phase. ${ }^{23}$, ${ }^{24}$ From turbidity measurements, Zhang et al. show that turbidity of PDADMA/PSS PECs drop with increasing salt concentration indicating dissolution. ${ }^{23}$ Works by Chollakup et al. and Li et al. also outline the ionic strength before and after preparation as an important phase-determining factor. ${ }^{18,25}$ Asides from salt, the polyelectrolyte mixing ratio, ${ }^{18,} 23$ mixing order, polyelectrolyte molecular weight, ${ }^{7,26}$ charge density, ${ }^{18,22}$ water content ${ }^{27}$, ${ }^{28}$ and, temperature. ${ }^{29}$

$$
\mathrm{Pol}^{+} \mathrm{Pol}_{s}^{-}+A^{-} \mathrm{M}^{+}{ }_{a q} \rightarrow \mathrm{Pol}^{+} A^{-}{ }_{a q}+\mathrm{Pol}^{-} \mathrm{M}^{+}{ }_{a q}
$$

Polyelectrolyte complexes have been described as glassy when dry and rubbery when wet, pointing to the fact that water is involved the plasticization of PECs. For neutral polymers, temperature has the plasticizing effect on a class of polymers known as thermoplastics. Whereas with PECs, temperature is shown to have a similar effect only in the presence of water molecules. Hence, PECs only show a glass transition when hydrated. With PECs, water salt and temperature have a plasticizing effect on PECs. 
Due to the plasticizing nature of salt on PECs, PECs have now been termed "saloplastics". This came at a time when PECs had been tagged unprocessable. PECs have then been developed into various forms such as capsules, micelles, gels and thin films. Now, a common variation of PECs available are polyelectrolyte multilayers (PEMs) prepared by a layer by layer technique.

\subsubsection{Polyelectrolyte multilayers (PEMs)}

As early as $1966,{ }^{30}$ multilayers prepared from colloidal mixtures by layer by layer were $(\mathrm{LbL})$ techniques were developed but have gained more attention by the works of Gero Decher in the 1990's. ${ }^{31}$ This came as a major advancement to adsorption techniques following Irving Langmuir monolayer adsorption theory. ${ }^{32,33}$ Polyelectrolyte multilayers (PEMs) are resulting thin films from the alternating LbL deposition of a polycation and polyanion characterized by stratified structures. ${ }^{31,34}$ Processing PECs by LbL allows for a control over nanoscale and microscale properties of the produced PEM. Hence, PEMs are highly tunable in nature and the structure, thickness, composition and dynamics can be altered by manipulating PE type, concentration, ionic strength, temperature and $\mathrm{pH} .{ }^{35-}$ 39 
a

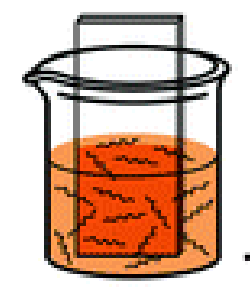

1) Polycation

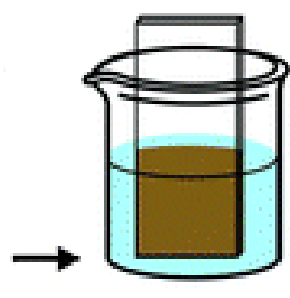

2) Water

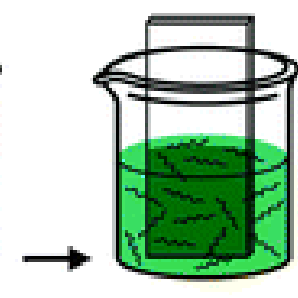

3) Polyanion

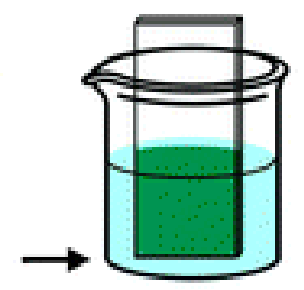

4) Water

b

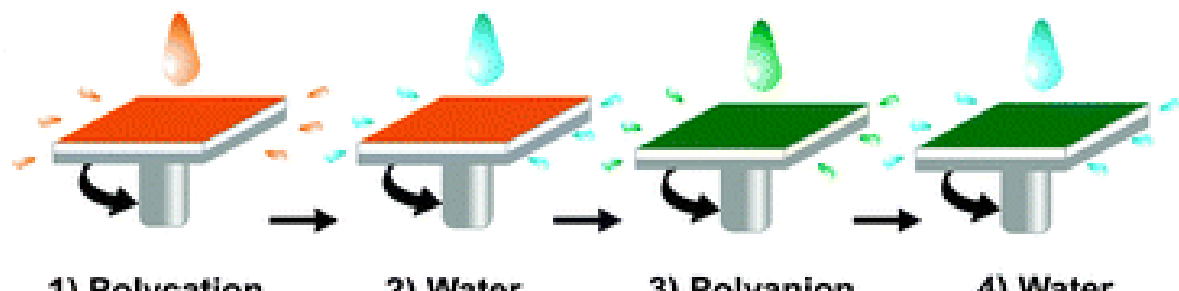

1) Polycation

2) Water

3) Polyanion

4) Water

c

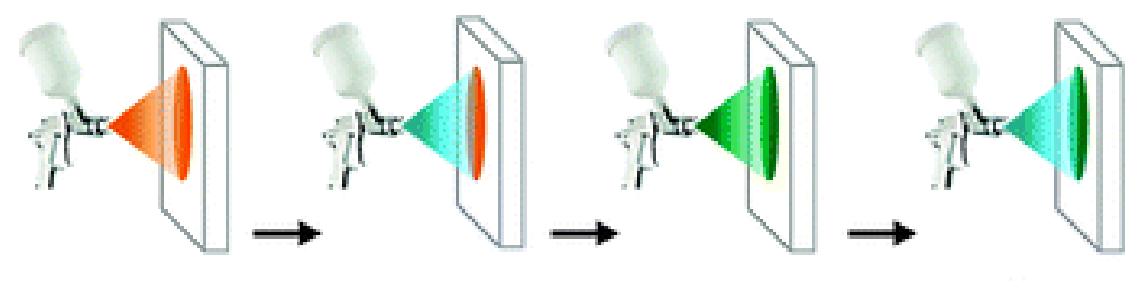

1) Polycation

2) Water

3) Polyanion

4) Water

Figure 1.3: Schematic representation of the processes used to fabricate polyelectrolyte multilayer films by LbL assembly. (a) Dipping LbL assembly. (b) Spin-assisted LbL assembly. (c) Spray-assisted LbL assembly. Multilayer films are formed by repeating steps 1 to 4 in a cycle fashion. Reproduced from Ref. [40] "Layer-by-layer assembly for rapid fabrication of thick polymeric films" by Li, Y., Wang, X., \& Sun, J., 2012. Chemical Society Reviews, 41(18), 5998-6009 with permission from The Royal Society of Chemistry.

LbL assembly involving the immersion of the intended substrate in each desired polymer solution followed by a rinse step to wash off all excess ions is the most common. 
However other techniques of LbL assemblies exist including spray and spin-assisted assemblies identified in Figure 1.3. ${ }^{40}$ Between each layer deposition, it is common to dry each layer but this step can and has been skipped especially when a mechanical robot is used for deposition.
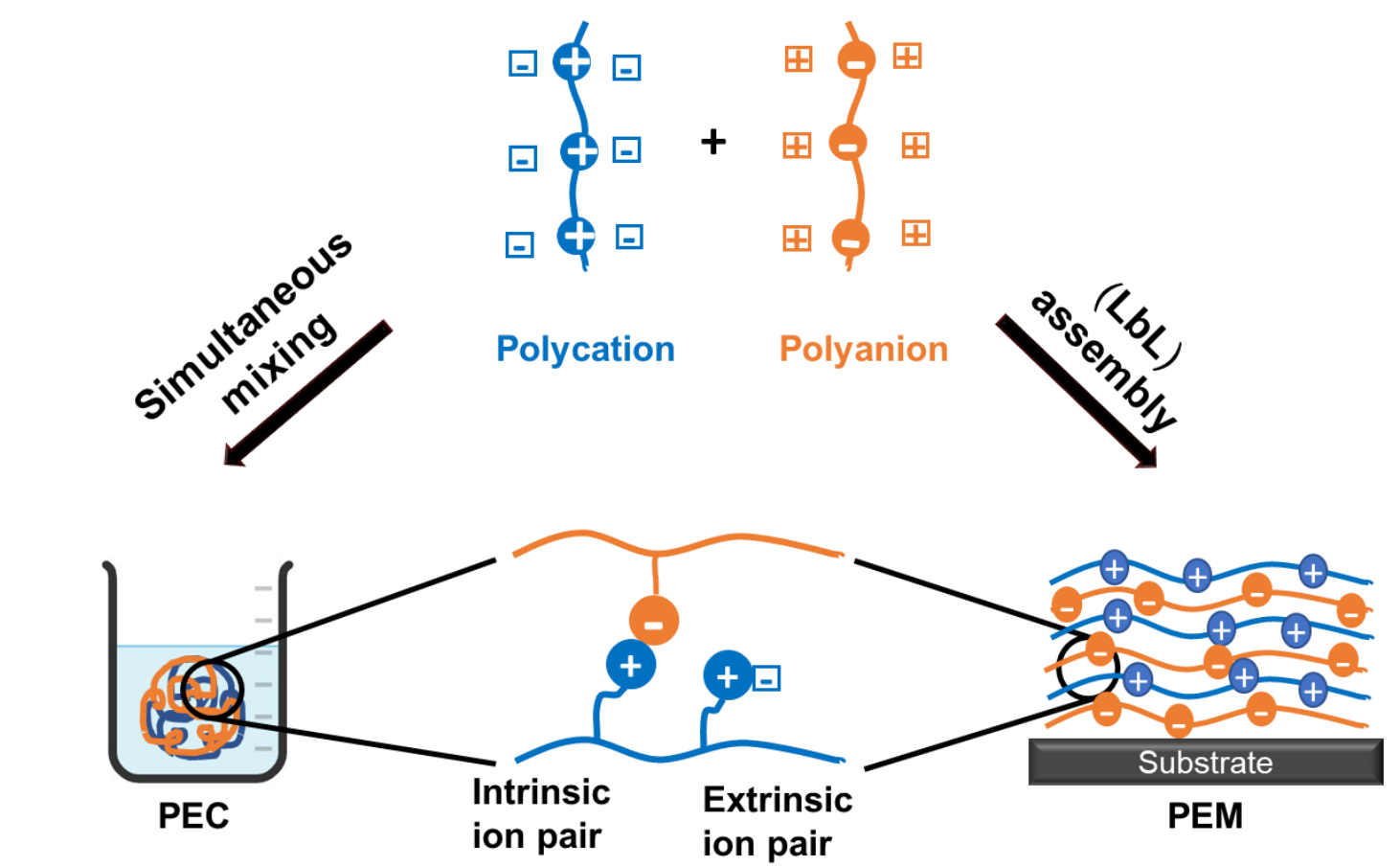

Figure 1.4: Comparison of polyelectrolyte complexes and multilayers

\subsubsection{Comparing PECs and PEMs}

Both PECs and PEMs are prepared from polyelectrolyte solutions with or without additional salt ions present. While PEC preparation involves quick mixing of both polyelectrolyte with one another, PEM preparation involves a simple LbL assembly technique. As shown by both Figure 1.4, while the macrostructure of both PECs and PEMs may the differ in appearance, they both comprise of two kinds of ion pairing, intrinsic and 
extrinsic. Both preparations are possible for practically every synthetic and natural polyelectrolyte available. However, in cases of extremely low charge density or excessive salt, complexation has been found to be difficult or simply impossible. ${ }^{22,41-43}$ Forces of complexation for both PECs and PEMs remain electrostatic attraction, hydrogen bonding and hydrophobic forces.

The composition of PECs often follows stoichiometry of preparation whereas for PEMs a non-stoichiometry often arises. ${ }^{44,} 45$ Phase profiles of PECs show that complexation may lead to the formation of solid precipitates, liquid-liquid phase separated coacervates, or soluble complexes. ${ }^{18,23}$ The most prominent condition for determining the produced phase is the salt concentration during preparation. ${ }^{23,24}$ On the other hand, the build-up of PEMs may either be in linear or exponential growth regime. ${ }^{38}$ A study ${ }^{46}$ by Izumrodov et al. brought together PEC phase behavior and PEM growth behavior. Results showed that at $0.3 \mathrm{M} \mathrm{NaCl}$ solution, both quaternized poly(4-vinylpyridine) (QPVP) and poly(methacrylic acid) (PMAA) PECs and PEMs yielded maximum mass of complex precipitate or film.

Binding energy and equilibration time have been shown to be dependent on the type of polyelectrolyte in use. ${ }^{47}$ Polyelectrolyte containing the sulfonate $\left(\mathrm{SO}_{3}{ }^{-}\right)$group for polyanions and amine $\left(\mathrm{NH}_{3}{ }^{+}\right)$group for polycations produce the strongest interchain interactions. ${ }^{47}$ When these two polyelectrolytes or a sulfonate-containing polyelectrolyte and most polycations mix, precipitation occurs as a result of thermodynamic stability fostered by kinetic restriction. For PEMs a short equilibration time would lead to the formation of films considered to be "kinetically-trapped". ${ }^{46,47}$ Longer equilibrium time 
would allow for mass loss. In the same study with QPVP/PMAA PEMs, thicker films were obtained when the deposition time was only 5 minutes than when it was 1 hour each. ${ }^{46}$

\subsubsection{Applications of PEMs}

Research on nanostructured polyelectrolyte assemblies continue to gain interest because of the wide range of possible applications. Self-healing properties of PEMs have been tapped into for use as protective coatings over corrosion effects or fire propagation. ${ }^{3 \text {, }}$ 4 Polymer-clay coatings made from branched polyethylenimine (BPEI) and montmorillonite (MMT) were demonstrated to have long-term environmentally-friendly anti-corrosion properties when used as pre-treatment for aluminum alloy. ${ }^{4}$ In another work, the same BPEI/MMT PEM has exhibited fire-retardation properties by of selfhealing. ${ }^{48}$

Interest in PEMs in medicine and pharmaceuticals is also on the rise. PEM capsules and micelles have been prepared and studied for use as targeted drug-delivery vehicles benefitting from PEM stimuli-responsiveness. ${ }^{49}$ The search for bio-smart coatings for body implants have also taken a turn to PEMs relying on the ability to be produced from natural polyelectrolytes and for the PEMs to remain stable at controlled conditions. ${ }^{6}$

\subsection{Fourier Transform Infrared Spectroscopy}

Fourier transform infrared (FTIR) spectroscopy is a material characterization technique commonly used in chemistry, physics, biology and engineering. It is used to identify functional groups in materials in the solid, liquid or gaseous state. ${ }^{50}$ Infrared (IR) spectroscopy techniques can be used to study a wide range of materials having covalent 


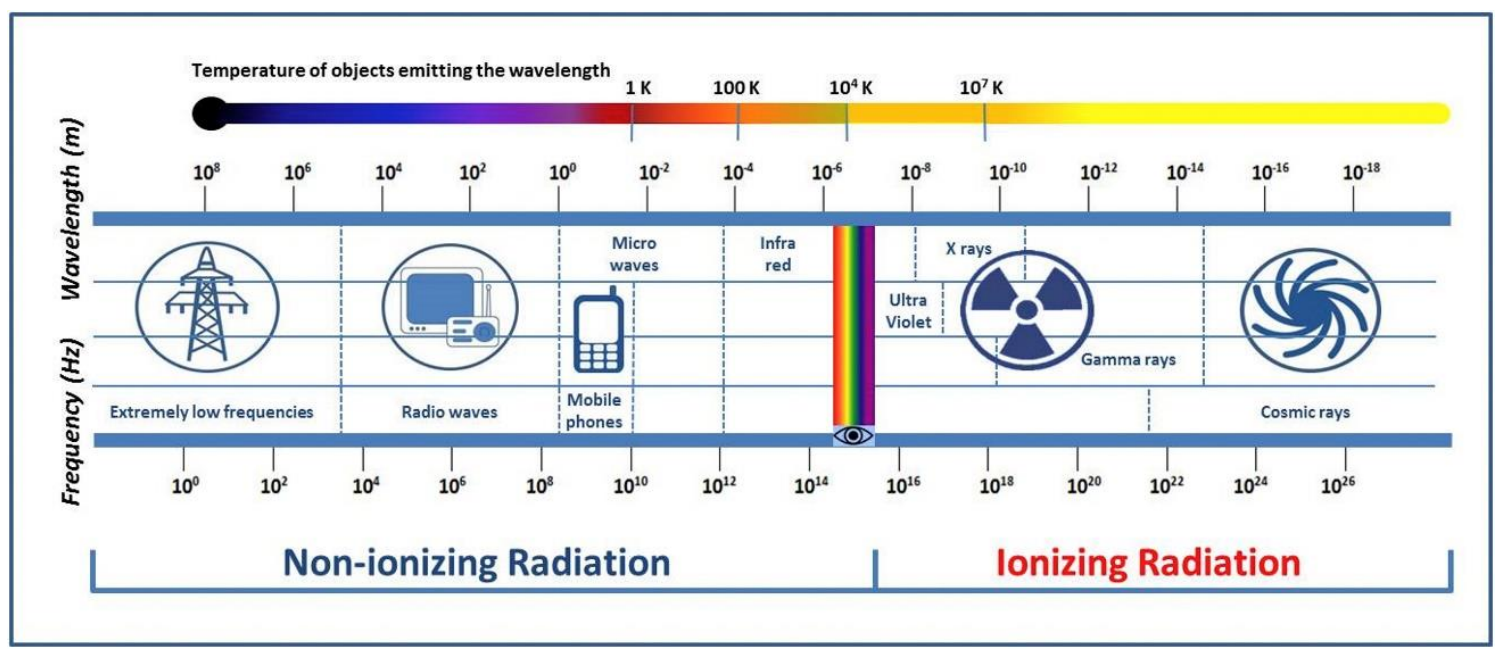

Figure 1.5: Electromagnetic spectrum. Figure reproduced with permission from ref [ $\left.{ }^{51}\right]$ "Biological effects of non-ionizing electromagnetic fields: Two sides of a coin" by Saliev, T., Begimbetova, D., Masoud, A.-R., \& Matkarimov, B., 2019. Progress in Biophysics and Molecular Biology, 141, 25-36 by Elseveir,

bonding using IR radiation. IR radiation has shorter wavelength and higher energy than microwave radiations but higher wavelength and lower energy than ultraviolet light.

FTIR spectroscopy operates by measuring the absorption or transmission of IR radiation by each bond in a molecule of the material. ${ }^{50}$ The result is presented as a broad spectrum of the $\%$ transmittance or absorbance plotted against the wavenumber $(\mathrm{v}) \mathrm{in}_{\mathrm{cm}}^{-}$ 1. This wavenumber is proportional to energy (E) and frequency (f) making it easier to interpret data off a spectrum.

$$
E=h f=h \frac{c}{\lambda}=h c v
$$


where h is Planck's constant $=6.62607004 \times 10^{-34} \mathrm{~m}^{2} \mathrm{~kg} / \mathrm{s}$

$\mathrm{c}$ is the speed of light $=3.0 \times 10^{8} \mathrm{~m} / \mathrm{s}$

$\lambda$ is the wavelength

From equation 1.3, we observe that higher wavenumber means higher frequency and larger energy and vice versa.

In order for a functional group to be identified by FTIR, it has to be IR active; that is, it should have a dipole moment. This enables the IR radiation to excite the covalent bond leading to bending (change in bond angles) or stretching (change in bond length) modes of the bond. As a result, single atoms are not IR active. A typical molecule studied, would have multiple IR active bonds which are not affected by each other. Each bond absorbs IR radiation at a particular frequency which produces the IR spectrum. While, two different molecules may have peaks at same wavenumbers, the overall spectrum cannot be the same. Each spectrum obtained acts as a fingerprint of the material's chemical structure. $^{52}$

There are four major types of FTIR spectroscopy, namely: transmission, attenuated total reflectance (ATR), specular reflectance and, diffuse reflectance FTIR. The 2 most common are transmission and ATR-FTIR for studies on polymers. Transmission FTIR reports resulting spectra as a plot of \% transmittance against wavenumber. Transmittance (T) is a ration of the transmitted IR beam to the incident IR beam. ${ }^{53}$ On the other hand, ATR-FTIR measures the absorbance of the IR beam using the total internal reflection property of a trapezoidal high refractive index crystal. ${ }^{54}$ When an IR beam passes through such a crystal an evanescent wave with a penetration depth peculiar to the material from 
which the crystal is made is formed.$^{54}$ In recent times, there now exist software that convert spectra obtained using ATR-FTIR to transmission spectra.

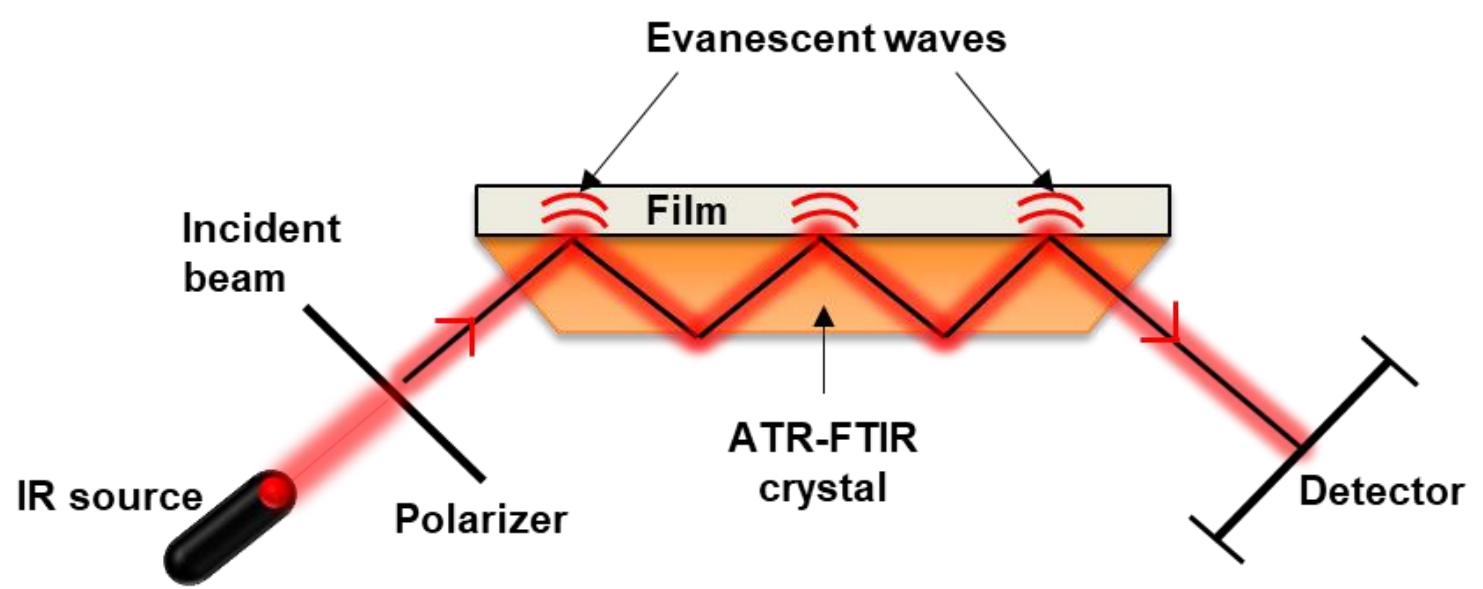

Figure 1.6: Working principle of ATR-FTIR

$$
T=\frac{I}{I_{o}}
$$

equation 1.4

where $\mathrm{T}$ is transmittance,

$\mathrm{I}_{\mathrm{o}}$ is the intensity of incident beam and,

I is the intensity of transmitted beam

Both transmittance and absorbance are related, ${ }^{53}$ and absorbance is calculated using the equation below:

$$
A=\log \left(\frac{I_{o}}{I}\right)=\log \left(\frac{1}{T}\right)
$$

equation 1.5 
where $\mathrm{A}$ is absorbance,

Another way of calculating absorbance is Beer's law which correlates absorbance to the concentration of molecules in sample as shown by the equation below:

$$
A=\varepsilon l c
$$

equation 1.6

where $\mathrm{A}$ is absorbance,

$\varepsilon$ is the molar absorptivity,

1 is the path length and,

$\mathrm{c}$ is the concentration of the sample

The height or area of a peak in an absorbance spectrum is proportional to concentration; therefore, Beer's law can be used to determine the concentrations of molecules in samples.

\subsection{Water in polyelectrolyte multilayers}

\subsubsection{Water content of polyelectrolyte multilayers}

A very prominent component present during the preparation of PEMs is water. Each polyelectrolyte is prepared into and aqueous solution and then after each deposition step involves a rinse with water or aqueous salt solution. It is no wonder that PEMs are typically hydrated when prepared and have to be heated to temperatures as high as $120^{\circ} \mathrm{C}$ to completely get rid of all water present. However, the water content of PEMs does vary with varying preparation conditions and even the post-assembly conditions. ${ }^{16,28,35,55-58}$

Water is a major defining factor in PEMs. Michaels, in 1965, observed that PEMs are brittle when dry and rubbery when wet. ${ }^{59}$ Huglin et al. ${ }^{60}$ also further discovered that 
in the absence of water, there is no thermal transition observed in PEMs, whereas in the presence of water, a "glass transition temperature" is observed. While some agree that this transition is a glass transition as the PEMs move from a glassy to a rubbery state, others argue against this proposition because the modulus in the "glassy" state is about 5 orders of magnitude lower than what is observable for regular polymers. ${ }^{28,29}$

Using FTIR spectroscopy, the obtained spectra for both PDADMA/PSS PEMs and PECs were mostly identical. ${ }^{36}$ For both PDADMA/PSS PEMs and PECs prepared in a 1:1 molar stoichiometry based on the polyelectrolyte repeat units, the broad peak at $3050 \mathrm{~cm}^{-}$ 1 indicated the presence of water. ${ }^{36}$ The IR sulfonate peak has often been used as a measure of PSS contribution to PEMs or PECs. ${ }^{36,61-63}$ Using sulfonate peak obtained from IR spectra of pure PSS in potassium bromide $(\mathrm{KBr})$ solution as a reference, the sulfonate peaks at $1050 \mathrm{~cm}^{-1}$ were integrated and recalibrated to obtain the amount of PSS present within the PEMs. The amount PSS here represents the amount of intrinsic ion pairs present. Similarly, the water content of the PEMs was obtained by integrating the $\mathrm{OH}$ stretch peak. The water content with respect to ion pairs was shown to increase from $0-$ 3 water molecules per ion pair as the number of layers increased from 0 to 15 layers at fixed $30 \% \mathrm{RH}$ and $25^{\circ} \mathrm{C} .{ }^{36}$

An important influence on the water content of PEMs is the outermost layer of the PEM. This is known as the odd-even effect. Previous studies suggest that this odd-even effect arises due to the loss of material from the PEM. But more recent studies show that the hydrophobicity or hydrophilicity of the charges on the terminating layer plays a major role in this odd-even effect. ${ }^{64}$ The charges in polyelectrolytes make most contributions to 
the hydrophilic behavior, without these charges, the hydrophobic behavior would be dominant. ${ }^{65}$ The most common way of observation is through thickness measurements. Neutron reflectometry studies ${ }^{65}$ showed that for PAH/PSS, PAH-terminated films yielded thinner films with lower total water volume fraction than PSS-terminated films.

Asides from the water content of freshly prepared PEMs, the water uptake into PEMs post-assembly has been investigated to better understand swelling and deswelling in PEMs. ${ }^{2,56,57,64,66-71}$ Post-treatment exposures at varying temperatures, salt solutions, relative humidity $(\mathrm{RH})$ and $\mathrm{pH}$ have been shown to induce swelling or shrinking of PEMs as would be discussed in later sections. Wong et al. showed, using PDADMA/PSS PEMs, that post-assembly swelling induced by changes in RH can be reversed. ${ }^{56}$ Swelling, water diffusion and mobility within PEMs have been studied using a vast range of technique including nuclear magnetic resonance (NMR) spectroscopy, ${ }^{69,}{ }^{72} \mathrm{X}$-ray reflectometry (XRR), ${ }^{16}$ neutron reflectometry (NR), ${ }^{16,39,56}$ ellipsometry, ${ }^{56}$ quartz crystal microbalance $(\mathrm{QCM})^{45}$ and FTIR. ${ }^{28,73}$

\subsubsection{Water micro-environments in PEMs}

While extensive research has been done to understand water uptake swelling as a whole, another group of researchers have embarked on the journey to identify the location of water molecules within the PEM films. ${ }^{16,74-76}$ This emanates from the differences identified in the techniques used to measure water content of the years. Water content of PEMs have been determined using various models. The earliest and simplest are based on changes in thickness or density of the PEMs as water content changes as described below; 
a. Thickness model assumes that all of the added water leads to a change in thickness (d) of the PEM.

$$
\emptyset_{\text {swell }}=\frac{d_{\text {swollen }}-d_{d r y}}{d_{\text {swollen }}}
$$

Where $\emptyset_{\text {swell }}$ is a measure of swelling water,

$d_{\text {swollen }}$ is the thickness of the swollen PEM and,

$d_{d r y}$ is the thickness of dry PEM

b. Density model accounts for changes in the scattering length density $(\mathrm{Nb})$ of the $\mathrm{PEM}^{77}$

$$
\emptyset_{\text {swell }}^{\prime}=\frac{N b_{\text {swollen }}-N b_{d r y}}{N b_{\text {water }}-N b_{d r y}}
$$

However more recently in 2010, Dodoo et al. reveal that there are discrepancies in the $\emptyset_{\text {swell }}$ and $\emptyset_{\text {swell }}^{\prime}$ values from both models for PDADMA/PSS. ${ }^{78}$ This suggests that there is some water which does not contribute to swelling thickness but influences the scattering length density of the PEMs. The inaccuracy of these two models lies in the existence of voids within the polyelectrolyte chains. This gave rise to the void model:

c. Void model accounts for voids present within polyelectrolyte chains ${ }^{78}$

$$
\emptyset_{\text {total }}=(1-x)\left(1-\emptyset_{\text {swell }}\right)+\emptyset_{\text {swell }}=\emptyset_{\text {void }}+\emptyset_{\text {swell }}
$$

Where $\mathrm{x}$ is the polymer fraction given by 


$$
x=\frac{N b_{\text {dry }}}{N b_{\text {water }}}-\frac{N b_{\text {swollen }}-\emptyset_{\text {swell }} N b_{\text {water }}}{\left(1-\emptyset_{\text {swell }}\right) N b_{\text {water }}}+1
$$

$\emptyset_{\text {total }}$ is the total amount of water present in PEM

$\emptyset_{\text {void }}$ is a measure of the void water present

$N b_{\text {water }}, N b_{\text {dry }}, N b_{\text {swollen }}$ are the scattering length densities of water, dry PEM and swollen PEM

Using neutron reflectometry, they identify the need to account for the voids in swelling because added water to the PEMs first fill up these voids before making any changes to the thickness. In this study, PDADMA/PSS PEMs were prepared in the presence of varying concentrations of $\mathrm{NaCl}, \mathrm{NaF}$, and $\mathrm{NaBr}$. Samples were then exposed to light and heavy water, $\mathrm{H}_{2} \mathrm{O}$ and $\mathrm{D}_{2} \mathrm{O}$ in vapor and liquid states. By observing changes in the scattering length density, they identify two types of water: void water and swelling water (Figure 1.7a). This was done by incorporating the $\emptyset_{\text {swell }}$ from the thickness model into the void model. They found that both $\mathrm{H}_{2} \mathrm{O}$ and $\mathrm{D}_{2} \mathrm{O}$ caused roughly equal amounts of swelling in the PEMs. The amount of swelling water increased with salt concentration and the order of the Hoffmeister series. Salt had no influence on the void water on the other hand.

Simpler techniques like ellipsometry have also proven the existence of both void and swelling water in PDADMA/PSS PEMs in the same fashion. ${ }^{16}$ In this case, in place of the scattering length density, permittivity $(\epsilon)$ which is directly proportional to the refractive index (n) of the PEMs was monitored. At $1 \%$ RH, PEMs are considered dry and contain voids which have the capacity to hold water (Figure $1.7 \mathrm{a}){ }^{74}$ The refractive index 


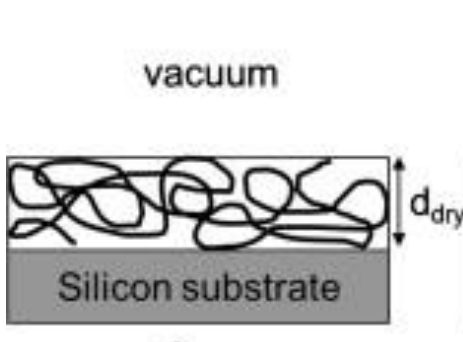

a

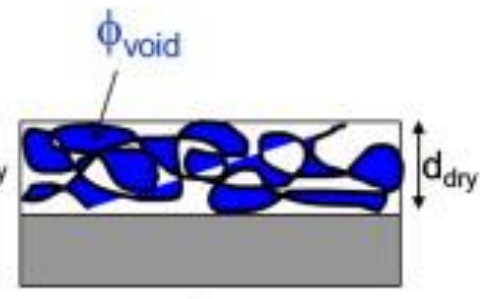

b

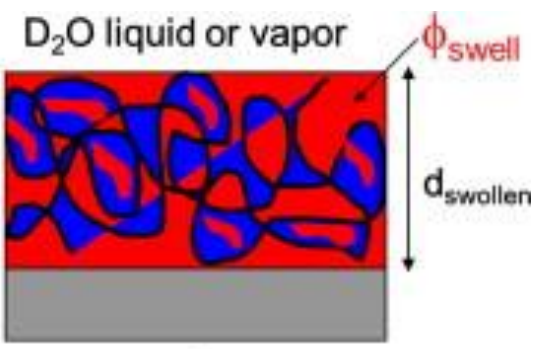

C

Figure 1.7: Swelling and void water in PEMs. Reprinted from reference [74] "About different types of water in swollen polyelectrolyte multilayers" by Koehler, R., Steitz, R., \& von Klitzing, R., 2014. Advances in Colloid and Interface Science, 207, 325-331 with permission from Elsevier.

of water ( $\left.\mathrm{n}_{\text {water }}\right)$ is 1.33 whereas that of a dry PEM ( $\mathrm{n}_{\text {dry PEM) is } 1.56 .}{ }^{16,56,79} 76$ As the PEMs become hydrated, the refractive index of the PEM (nPEM) gradually decreases. While the void model provided a new insight to the microenvironments of water in PEMs, the model is only fully successful at $\mathrm{RH}>20 \%$. Below $20 \% \mathrm{RH}$, the measured data begins to diverge from the fit. As a result, this more recent study in 2018 probes further with an extended void model. The extended void model now accounts for influence of air-water exchange within the voids on the permittivity of the PEM.

d. Extended void model which accounts for the voids between polyelectrolyte chain as well as exchange between air and water in voids

$$
\epsilon_{\text {swollen }}=S \cdot \epsilon_{\text {water }}+(1-S) \cdot\left[x \cdot \epsilon_{\text {poly }}+(1-x) \cdot\left(a \cdot \epsilon_{\text {water }}+(1-a) \cdot \epsilon_{\text {air }}\right)\right]
$$

Where $\mathrm{S}$ is the swelling water as in thickness model

$\epsilon_{\text {water }}, \epsilon_{\text {air }}$, and $\epsilon_{\text {poly }}$ is the permittivity of water, air and pure polymer 
$\mathrm{x}$ is the polymer fraction and

a describes the air- water exchange

$$
a=\begin{array}{ll}
b \cdot S & \text { for } S<0.08 \\
1 & \text { for } S \geq 0.08
\end{array}
$$

$\mathrm{b}$ is a fitting parameter

Results show that at $\mathrm{RH}<30 \%$, air-water exchange still takes place and the voids are not entirely filled with water. This is evident as the value of the parameter, a increases up to $30 \% \mathrm{RH}$ before stabilizing. Though this study claims to provide the first model of airwater exchange, they also admit that possible errors may arise in the model as the void sizes change with humidity.

Another approach is by studying the thermal properties of water within the PEM films. One study using modulated differential scanning calorimetry (MDSC), revealed that by monitoring the freezing temperature of water at different PEM hydration levels from $18-30 \mathrm{wt} \%$ water, the state of water can be identified. ${ }^{75}$ Characteristic freezing temperature of water is at $273 \mathrm{~K}\left(0^{\circ} \mathrm{C}\right)$. Three water microenvironments were identified in this study, namely: freezing free water, freezing bound water, and non-freezing bound water. Freezing free water is water which still freezes at the normal water freezing temperature of $273 \mathrm{~K}$. Freezing bound water is water which freezes at a temperature lower than $273 \mathrm{~K}$ indicating some influence from polyelectrolyte ion pairing. Lastly, non-freezing bound water is water which shows no sign of freezing within the films. These are considered to be very tightly bound to ion pairs. For the studied range, no freezing free water was found. 


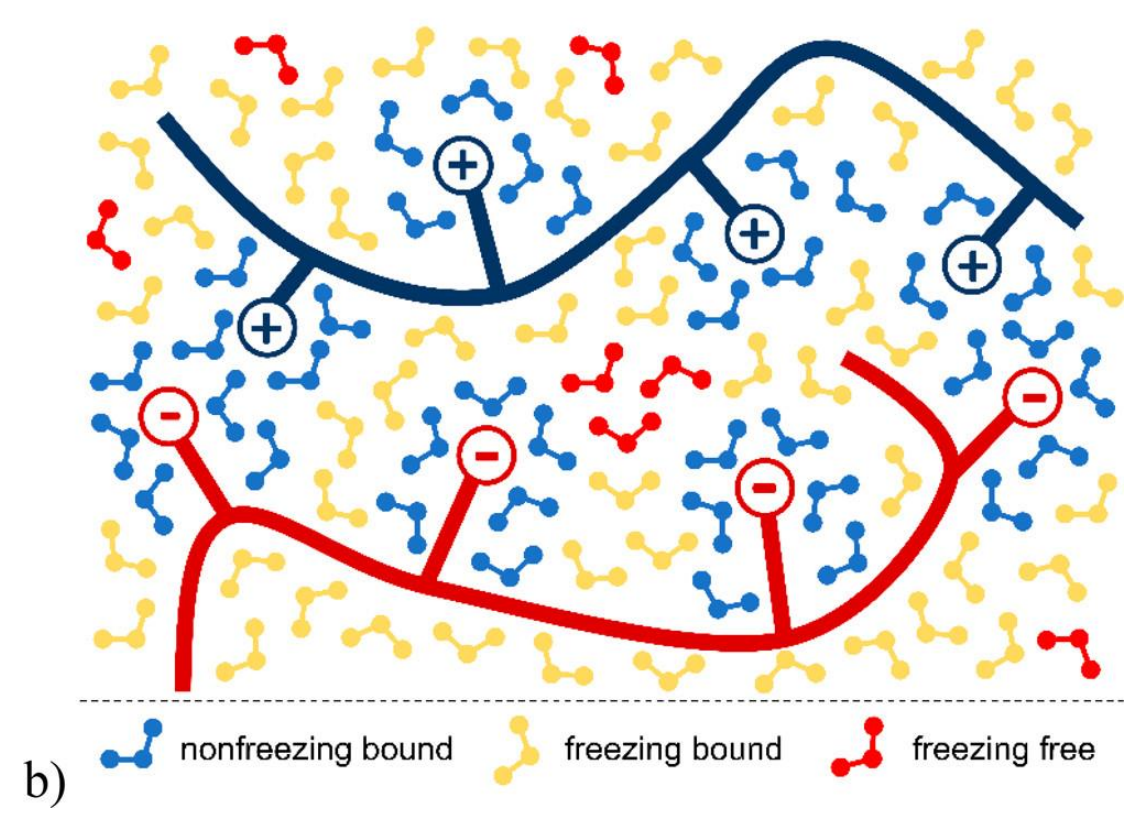

Figure 1.8: Freezing free, freezing bound and non-freezing bound water. Reprinted with permission from [75] "Hydration and Temperature Response of Water Mobility in Poly(diallyldimethylammonium)-Poly(sodium 4-styrenesulfonate) Complexes" by Batys, P., Zhang, Y., Lutkenhaus, J. L., \& Sammalkorpi, M., 2018. Macromolecules, 51(20), 8268-8277. Copyright (2018) American Chemical Society https://pubs.acs.org/doi/full/10.1021/acs.macromol.8b01441

\subsubsection{Effects of ionic strength on water content of PEMs}

The presence of salt in the contacting polyelectrolyte solutions during build-up cause charges to be extrinsically-compensated as opposed to being intrinsically compensated in the absence of salt ions. Also, when PEMs are exposed to salt solutions after preparation, rearrangement of ion pairing occurs. This leads to swelling and 
smoothening of PEMs. By swelling, we expect that the introduction of salt ions into the PEMs would increase its hydrophilicity and in turn attract more water to itself. This process has been termed salt annealing or salt doping. ${ }^{64}$

Swelling ratio of PDADMA/PSS films calculated as the ratio between wet and dry thickness of the films were observed to study swelling as a function of salt concentration. Beyond 2.0 M NaCl, PDADMA/PSS films began to disintegrate, whereas this critical concentration for PAA/PDADMA films is as low $0.3 \mathrm{M} \mathrm{NaCl} .{ }^{64}$ This shows that the extent to which salt influences extend is also control by the type of polyelectrolyte in question and its charge density and hydrophobicity. At very low salt concentration $(\sim<0.25 \mathrm{M})$, the films experience deswelling probably as a result of osmotic effects. However, with the addition of more salt $(>0.25 \mathrm{M})$, the films begin to regain its thickness, achieving its initial thickness at $\sim 0.5 \mathrm{M}$. Above $0.5 \mathrm{M}$, swelling truly picks up until $2.0 \mathrm{M} \mathrm{NaCl}$.

Using neutron reflectometry, effects of salt on water microenvironments, swelling and void water, was investigated. ${ }^{78}$ For 6 layer pairs of PDADMA/PSS films prepared in the absence of salt, the reported thicknesses for $0.1 \mathrm{M}, 0.25 \mathrm{M}$ and $0.5 \mathrm{M}$ were $144 \pm 1 \AA$, $267 \pm 6 \AA$, and $481 \pm 3 \AA$, respectively. However, as total water content increases, only the amount of swelling water increases while void water actually decreases. This may be attributed to the reduction in voids as polymer chains become more interdigitated. As confirmed by both studies described, salt ions enhance mixing of polymer chains within the PEMs and in turn promote smoothening of film surface. 


\subsubsection{Effects of temperature on water content of PEMs}

The effect temperature has on PEMs has widely studied to gain full understanding of PEMs which may be incorporated into high temperature applications. Polymer chains within PEMs respond to changes in temperature. At higher temperature, it is assumed that polymer chains gain energy, increase polymer conformation dynamics, thereby increasing the distance between charges. ${ }^{80-82}$ PSS/PAH PEMs prepared at an elevated temperature of $55^{\circ} \mathrm{C}(100 \mathrm{~nm}$ at 20 layers) showed an accelerated growth rate compared to those prepared at $19{ }^{\circ} \mathrm{C}(100 \mathrm{~nm}$ at 36 layers $) .{ }^{82}$ However, post-assembly thermal treatments of PEMs have some influence on thickness, ${ }^{80}$ capsule diameter (for PEM capsules), ${ }^{81}$ surface roughness ${ }^{80}$, and water content.

Observable changes in thickness are a function of swelling and chain conformations. The relationship between temperature and is a two-way relationship. While the temperature of preparation or post-assembly temperature influences the water content of the films, water content of the films in turn influence the thermal transition temperature of films. ${ }^{27,75,83}$ 


\section{CHAPTER II}

\section{EXPERIMENTAL SECTION ${ }^{1}$}

\subsection{Materials}

Poly(diallyldimethylammonium chloride) (PDADMA, $\mathrm{M}_{\mathrm{w}}=200,000-350,000$ $\mathrm{g} / \mathrm{mol}, 20 \mathrm{wt} \%$ solution), polystyrene sulfonate (PSS, $\mathrm{M}_{\mathrm{w}}=500,000 \mathrm{~g} / \mathrm{mol}$ ) were purchased from Polysciences, Inc. Deuterium oxide $\left(\mathrm{D}_{2} \mathrm{O}\right)(99.8 \%$ deuterium $)$ was purchased from Tokyo Chemical Industries Co. and sodium chloride $(\mathrm{NaCl})$ was purchased from Sigma-Aldrich. The substrate used was a $45^{\circ}$ angle Zinc Selenide $(\mathrm{ZnSe})$ crystal purchased from Specac Ltd.

\subsection{Methods}

\subsubsection{Solution preparation}

Each PDADMA and PSS solution in water was prepared at a concentration of 1 $\mathrm{g} / \mathrm{L}$. For layer-by-layer assembly, the salt concentration of both polyelectrolyte solutions and rinsing solutions were $0.5 \mathrm{M} \mathrm{NaCl}$. HOD mixture was prepared using $5 \%$ by volume of $\mathrm{D}_{2} \mathrm{O}\left(4.52 \mathrm{~mol} \% \mathrm{D}_{2} \mathrm{O}\right.$ in $\left.\mathrm{H}_{2} \mathrm{O}\right)$ with $95 \%$ Milli-Q deionized $\mathrm{H}_{2} \mathrm{O}$ to make $\mathrm{NaCl} \mathrm{HOD}$ solutions used for ATR-FTIR spectroscopy measurements.

\footnotetext{
${ }^{1}$ Adapted from Ref. 66. Eneh, C. I.; Bolen, M. J.; Suarez-Martinez, P. C.; Bachmann, A. L.; Zimudzi, T. J.; Hickner, M. A.; Batys, P.; Sammalkorpi, M.; Lutkenhaus, J. L., Fourier transform infrared spectroscopy investigation of water microenvironments in polyelectrolyte multilayers at varying temperatures. Soft Matter 2020, 16 (9), 2291-2300. with permission from The Royal Society of Chemistry.
} 


\subsubsection{Layer by Layer (LbL) assembly}

ZnSe crystal substrate was cleaned using Milli-Q water then acetone, followed by air-drying to remove any residual solvent. $2.5 \mathrm{M} \mathrm{NaCl}$ was used to remove any residual deposits on the crystal. LbL assembly of the polyelectrolytes was performed using an automated HMS slide stainer from Carl Zeiss, Inc. at room temperature. The ZnSe crystal was placed in a basket with the surface to be tested exposed. One layer of PDADMA on ZnSe crystal was made by immersing the crystal in 1g/L PDADMA solution for $15 \mathrm{~min}$., followed by three rinses in $0.5 \mathrm{M} \mathrm{NaCl}$ with agitation for $2 \mathrm{~min}$., $1 \mathrm{~min}$., and $1 \mathrm{~min}$. Similarly, a layer of PSS was deposited by repeating the same sequence. Dry thickness of 70 layer-pairs of PDADMA/PSS films, measured using a profilometer, was determined to be $2.5 \mu \mathrm{m}$. 70 layer-pair films were thus prepared for all measurements to ensure that the film thickness was greater than the ZnSe penetration depth of the ATR-FTIR evanescent wave $(0.71-0.80 \mu \mathrm{m}$ for $2400-2700 \mathrm{~cm}-1)$. PEM films dried at ambient conditions overnight. 


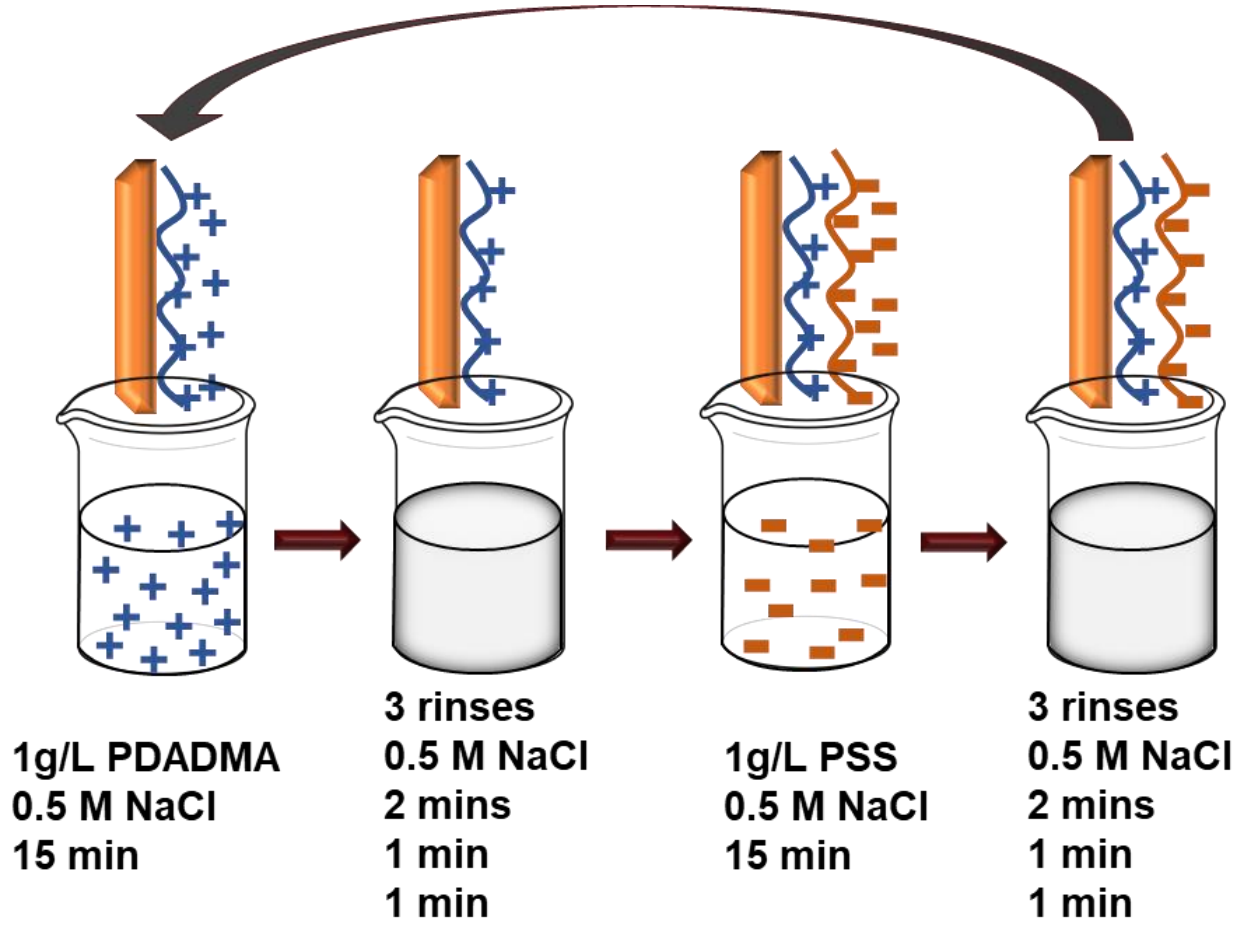

Figure 2.1: Schematic of layer by layer assembly of PDADMA/PSS films

\subsubsection{ATR-FTIR spectroscopy}

ATR-FTIR spectroscopy was performed using a Nicolet 6700 FTIR spectrometer from Thermo Scientific. A ZnSe crystal background spectrum was taken on a clean crystal under $\mathrm{CO}_{2}$-free conditions over a temperature range of $35-70{ }^{\circ} \mathrm{C}$ increasing by $5{ }^{\circ} \mathrm{C}$ temperature increments to assess the effect of temperature on background spectra. The background spectra were independent of temperature, thus the background spectrum at 35 ${ }^{\circ} \mathrm{C}$ was chosen as the representative reference spectrum for all subsequent experiments. Each spectrum was recorded with a resolution of $4 \mathrm{~cm}^{-1}$ with 32 scans over the range of $400-4000 \mathrm{~cm}^{-1}$. A low hydration ATR-FTIR spectroscopy measurement was performed 
on each PEM using $100 \mu \mathrm{L}$ of varying concentrations of $\mathrm{NaCl} \mathrm{HOD}$ pipetted into FTIR stage. Following the same procedure, measurements were performed over the same temperature range on each PEM fully immersed in $\sim 850 \mu \mathrm{L}$ of $\mathrm{NaCl}$ HOD solutions.

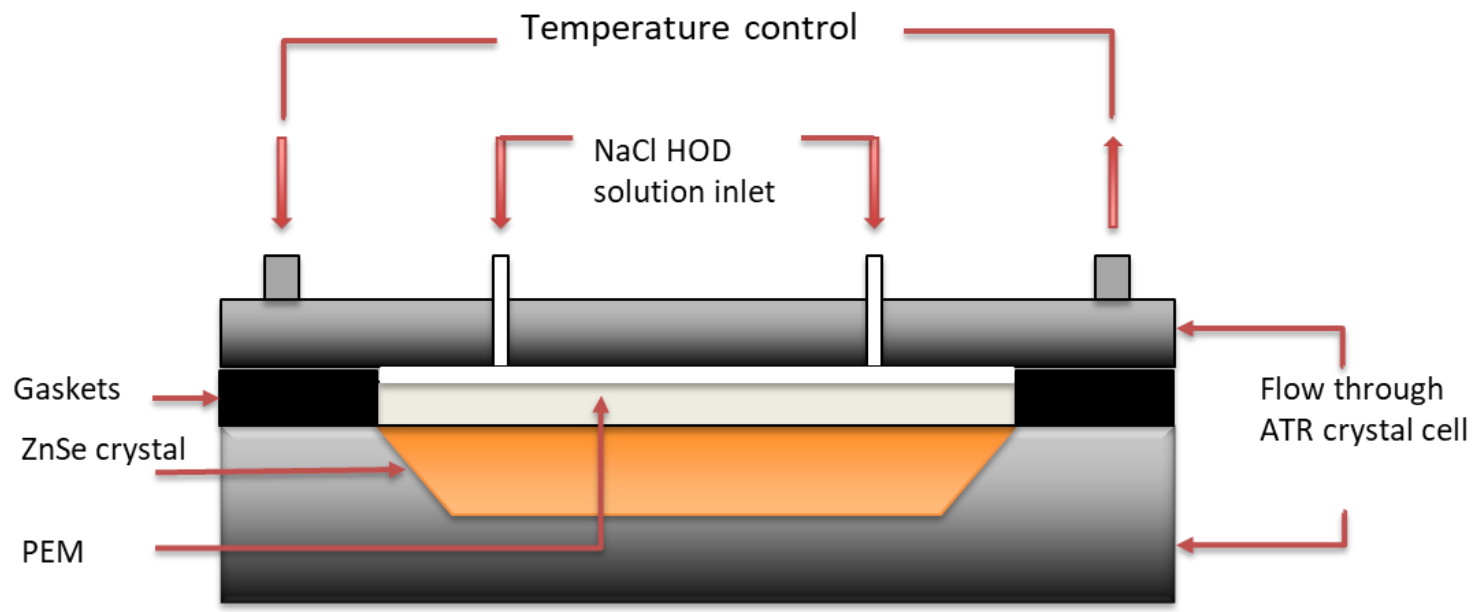

Figure 2.2: FTIR experimental set-up

\subsubsection{Non- Condon effects}

For this reason, all OD peaks were corrected for non-Condon effects and analyzed using a previously published three-population deconvolution method ${ }^{84}$ with Origin 8.0 Pro.

$$
\begin{array}{cr}
\frac{\mu}{\mu_{g}}=31.27-\left(1.09 \times 10^{-2} \mathrm{~cm}\right) \omega & \text { equation } 2.1 \mathrm{a} \\
A_{\text {corrected }}=\frac{A_{\text {fit }}}{\mu^{2}} & \text { equation } 2.1 \mathrm{~b}
\end{array}
$$


where $\mu$ is the transition dipole at each wavenumber and where $\mu_{g}$ and $\omega$ are the gas-phase transition dipole and frequency of the vibration respectively.

\subsubsection{FTIR analysis}

All spectra were processed using Omnic software and were baselined by fixing the absorbance at the end points of the wavelength region $\left(400-4000 \mathrm{~cm}^{-1}\right)$. The examined range represents the OD asymmetrical stretch peak and the spectrum was deconvoluted to show the following water microenvironments: low and high frequency and bulk water peaks. The high frequency component corresponds effectively to stronger PE-water interactions (weaker hydrogen bonds) and the low frequency components to weaker PEwater interactions (stronger hydrogen bonds) ${ }^{85}$ Therefore, the water molecules with high frequency OD stretching can be identified as strongly bound to the polyelectrolyte. Simply put,

- $\quad$ High frequency water $=$ tightly bound to $\mathrm{PE}$ ion pair $=$ weak $\mathrm{H}_{2}$ bonds

- Low frequency water $=$ loosely bound to $\mathrm{PE}$ ion pair $=$ stronger $\mathrm{H}_{2}$ bonds

- Bulk water $=$ water free of PE ion pair influence

Figure 2.3a shows a sample of a deconvoluted OD stretch peak showing the three identified peaks. Figure $2.3 \mathrm{~b}$ is an illustration of these water microenvironments located around ion pairs. 

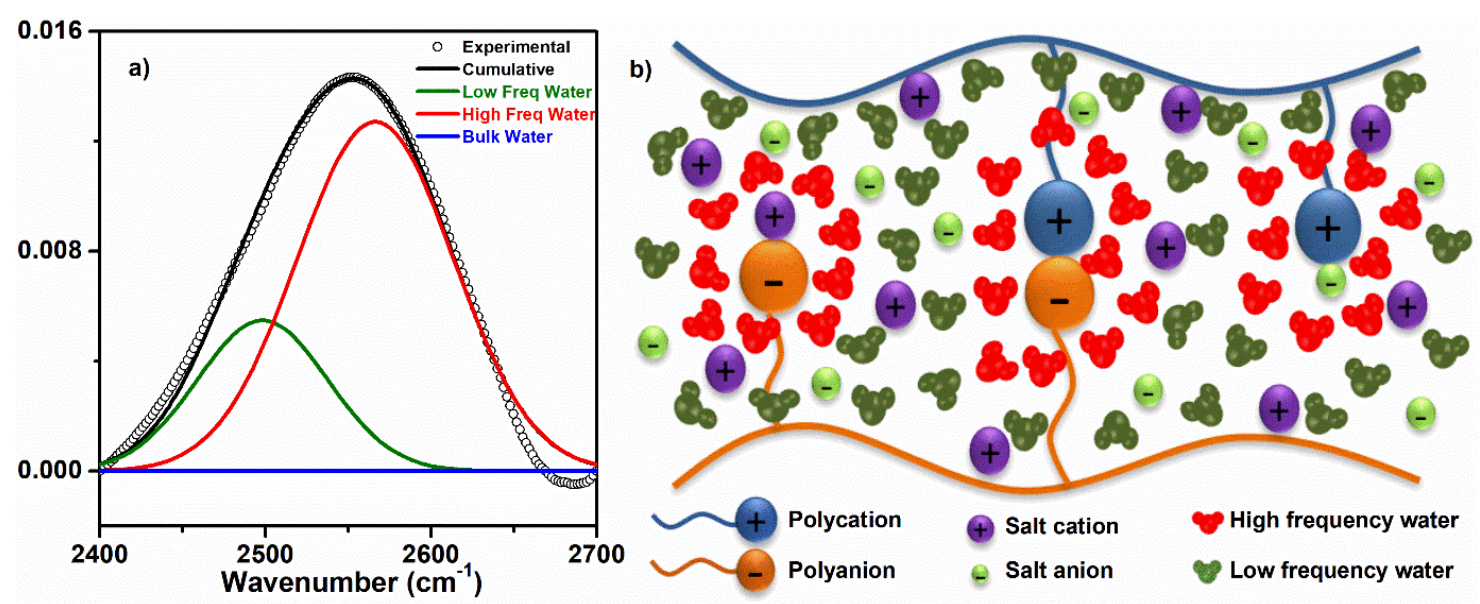

Figure 2.3: (a) A sample spectrum showing deconvolution of the OD asymmetrical stretching region from 2400 to $2700 \mathrm{~cm}^{-1}$ of PDADMA/PSS PEM immersed in $0.50 \mathrm{M}$ $\mathrm{NaCl} \mathrm{HOD}$ solution. The three peaks (in blue, green, and red) correspond to populations of HOD in different states of association within the surrounding environment: bulk water, low frequency water, and high frequency water. (b) A visual representation of the water microenvironments and their distribution around both intrinsic and extrinsic ion pairs showing low frequency and high frequency water.

First, the low and high frequency peak shapes were determined by examining a PEM under low hydration conditions; a three-peak fit was performed by fixing the bulk water peak center and full width half $\max (\mathrm{FWHM})\left(2509 \mathrm{~cm}^{-1}\right.$ and $170 \mathrm{~cm}^{-1}$, respectively) ${ }^{86,87}$ while allowing the other peak shapes (peak center and FWHM) to vary. For fully immersed samples, the bulk water peak intensity was then unconstrained, while fixing the high frequency peak shape (center and FWHM) and the low frequency peak center. The low frequency peak's FWHM was left unconstrained because as hydration 
increases, the low frequency peak's FWHM will potentially change due to a broadening of the distribution of states within this population. ${ }^{88}$ The fits were iterated until their cumulative sum of the least squares difference was minimized. In examining all of the data, the deconvolutions were essentially reduced to two peak fittings due to the very small contribution from the bulk water peak. 


\section{CHAPTER III}

\section{RESULTS AND DISCUSSION ${ }^{2}$}

\subsection{Background FTIR spectra}

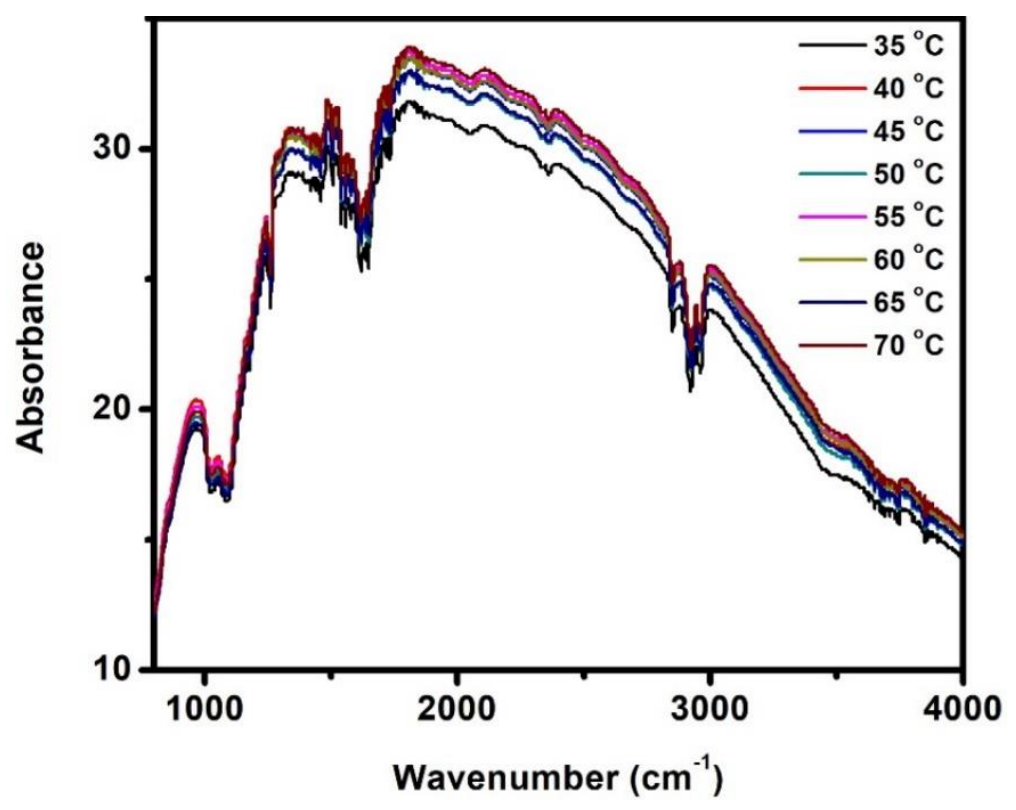

Figure 3.1: FTIR spectra of bare ZnSe crystal at different temperatures $\left(35-70{ }^{\circ} \mathrm{C}\right.$ at 5 ${ }^{\circ} \mathrm{C}$ intervals)

In order to obtain the true FTIR spectrum of a material, we baselined the initial FTIR spectrum obtained for the sample material with a reference spectrum. Here, we use the spectrum obtained from the bare $\mathrm{ZnSe}$ crystal in air as the required background

${ }^{2}$ Adapted from Ref. 67. Eneh, C. I.; Bolen, M. J.; Suarez-Martinez, P. C.; Bachmann, A. L.; Zimudzi, T. J.; Hickner, M. A.; Batys, P.; Sammalkorpi, M.; Lutkenhaus, J. L., Fourier transform infrared spectroscopy investigation of water microenvironments in polyelectrolyte multilayers at varying temperatures. Soft Matter 2020, 16 (9), 2291-2300. with permission from The Royal Society of Chemistry. 
spectrum. Figure 3.1 shows the FTIR spectra of the bare ZnSe crystal obtained at different temperatures from $35-70{ }^{\circ} \mathrm{C}$ at $5{ }^{\circ} \mathrm{C}$ intervals in order to get a baseline for each temperature used in the entire study. However, we observe that temperature has minimal influence on the background spectra as seen in Figure 3.1. As a result, we proceed with the rest of the experiments using the background spectrum for bare $\mathrm{ZnSe}$ crystal at $35^{\circ} \mathrm{C}$ as a baseline.

\subsection{OD stretch peak}

Taking into consideration the difficulties associated with analyzing $\mathrm{OH}$ stretch peaks, which have overlapping bands, ${ }^{89}$ the OD stretch peak instead was used to study water interactions in PDADMA/PSS multilayer systems. The presence of an OD stretch (2400 $-2700 \mathrm{~cm}^{-1}$ ) peak is verified in Figure 3.2 upon addition of $5 \% \mathrm{v} / \mathrm{v} \mathrm{D}_{2} \mathrm{O}$ to $\mathrm{H}_{2} \mathrm{O}$.

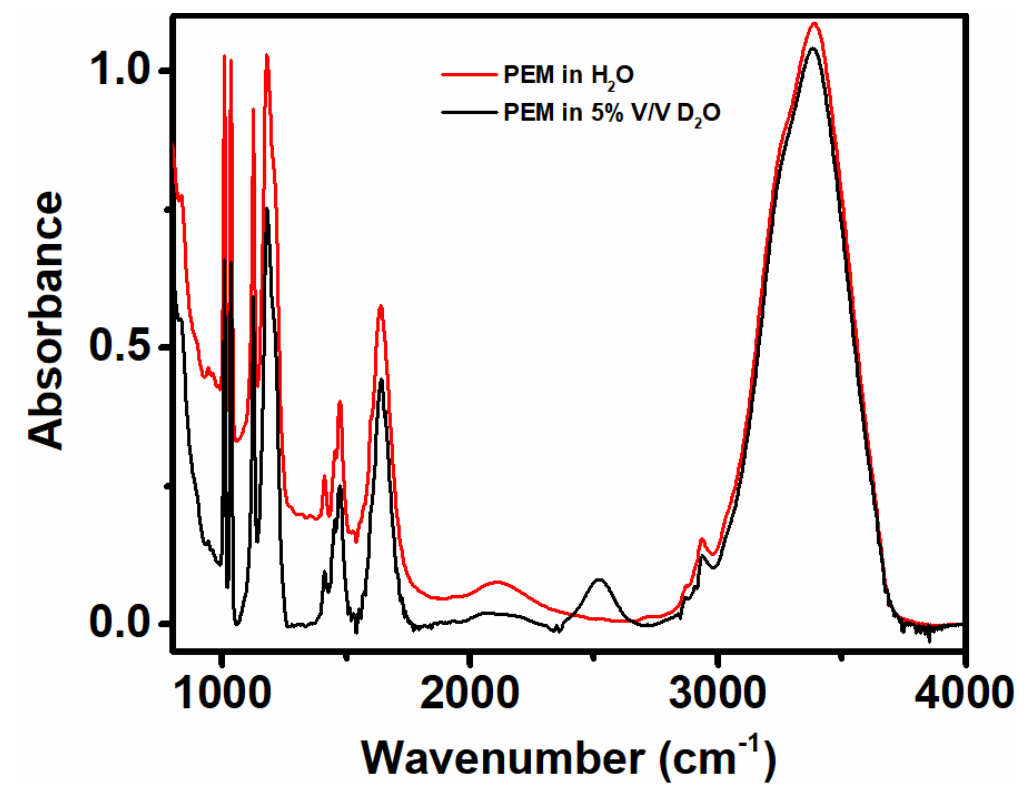

Figure 3.2: Comparison of $5 \mathrm{wt} \% \mathrm{D}_{2} \mathrm{O}$ in $\mathrm{H}_{2} \mathrm{O}$ showing lack of interfering peaks in the OD region $\left(2700-2400 \mathrm{~cm}^{-1}\right)$. Spectra were not corrected for non-Condon effects. 


\subsection{Non-Condon correction of OD stretch peak}

In spectroscopy, non-Condon effects are explained by the dependence of the vibrational transitional dipole moment of a molecule on the rotational and translational coordinates of all the molecules in the liquid. ${ }^{90}$ These non-Condon effects are more pronounced in strongly hydrogen-bonded systems like dilute $\mathrm{HOD}$ in $\mathrm{H}_{2} \mathrm{O} .{ }^{90}$ The bond dipole derivative of an $\mathrm{OH}$ stretch is dependent on the hydrogen-bonding environment. ${ }^{90}$ Figure 3.3 shows the resulting spectra from non-Condon correction using equations $2.1 \mathrm{a}$ and $\mathrm{b}$ in contrast to the raw spectrum obtained.

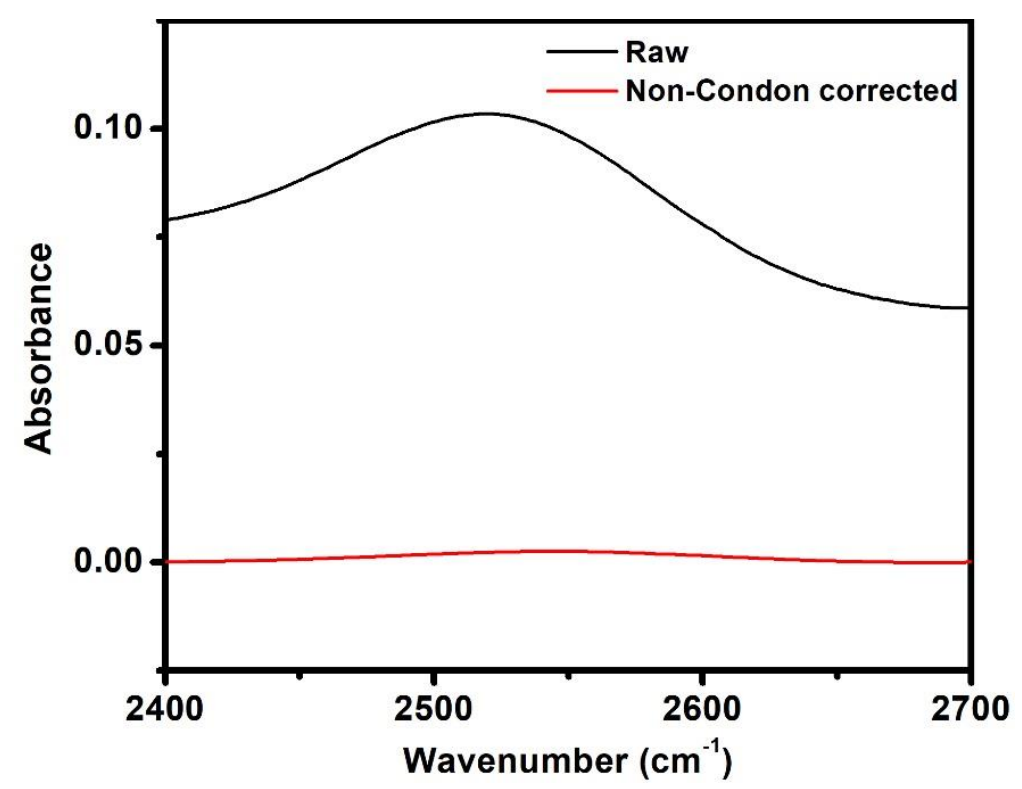

Figure 3.3: Shape comparison disregarding peak height shows the shift of the overall peak maximum towards higher frequencies when spectra are corrected for non-Condon effects as described in the materials and methods section. Uncorrected left Y-axis, Non-Condon corrected right Y-axis. Data corresponds to $0.25 \mathrm{M} @ 35{ }^{\circ} \mathrm{C}$ 


\subsection{FTIR spectra of PDADMA/PSS PEMs}

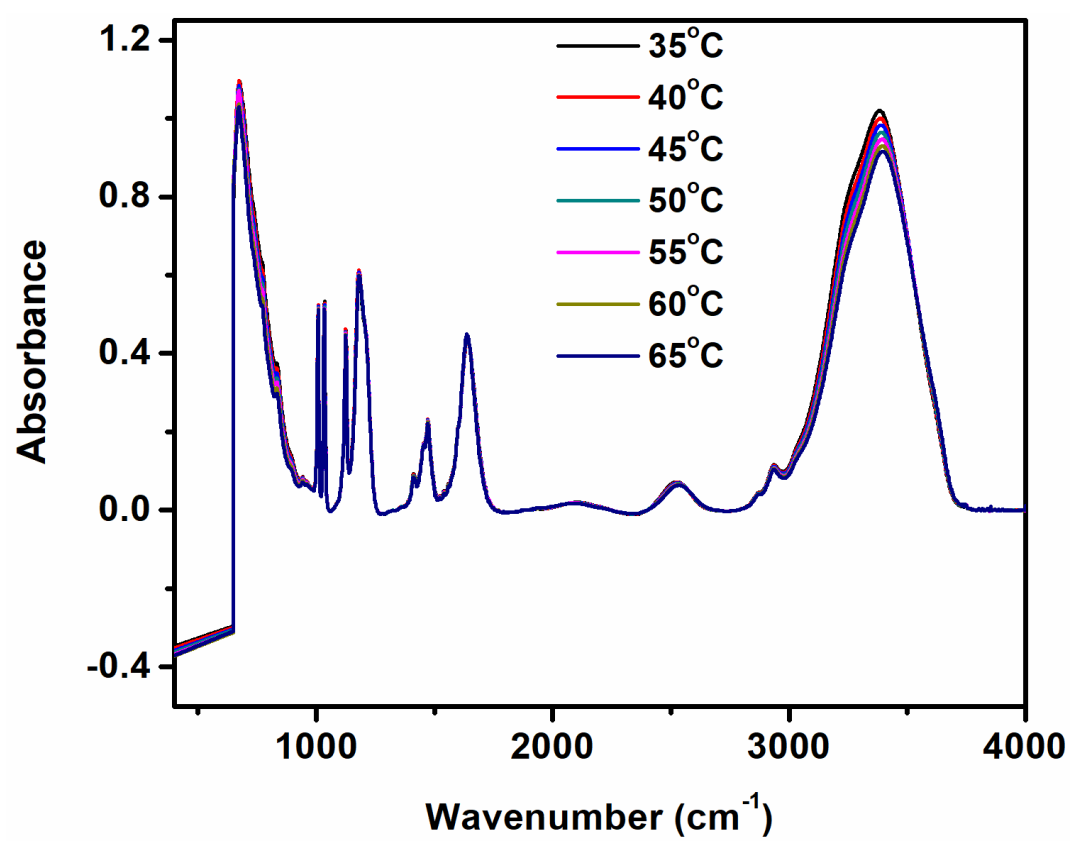

Figure 3.4: Set of baselined FTIR spectra $\left(400-4000 \mathrm{~cm}^{-1}\right)$ for a 70 -layer pair PDADMA/PSS film assembled in $0.50 \mathrm{M} \mathrm{NaCl}$ solution and immersed in $0.50 \mathrm{M} \mathrm{NaCl}$ HOD solution at temperatures from $35-65^{\circ} \mathrm{C}$

Figure 3.4 shows FTIR spectra over the range of $400-4000 \mathrm{~cm}^{-1}$ for a film immersed in $0.5 \mathrm{M} \mathrm{NaCl} \mathrm{HOD.} \mathrm{The} \mathrm{full} \mathrm{spectra} \mathrm{comprise} \mathrm{of} \mathrm{the} \mathrm{polymer} \mathrm{fingerprint} \mathrm{region}$ from $1000-1500 \mathrm{~cm}^{-1}$, the OD stretch region from $2400-2700 \mathrm{~cm}^{-1}$ and, the $\mathrm{OH}$ stretch peak from $2800-3800 \mathrm{~cm}^{-1}$. 


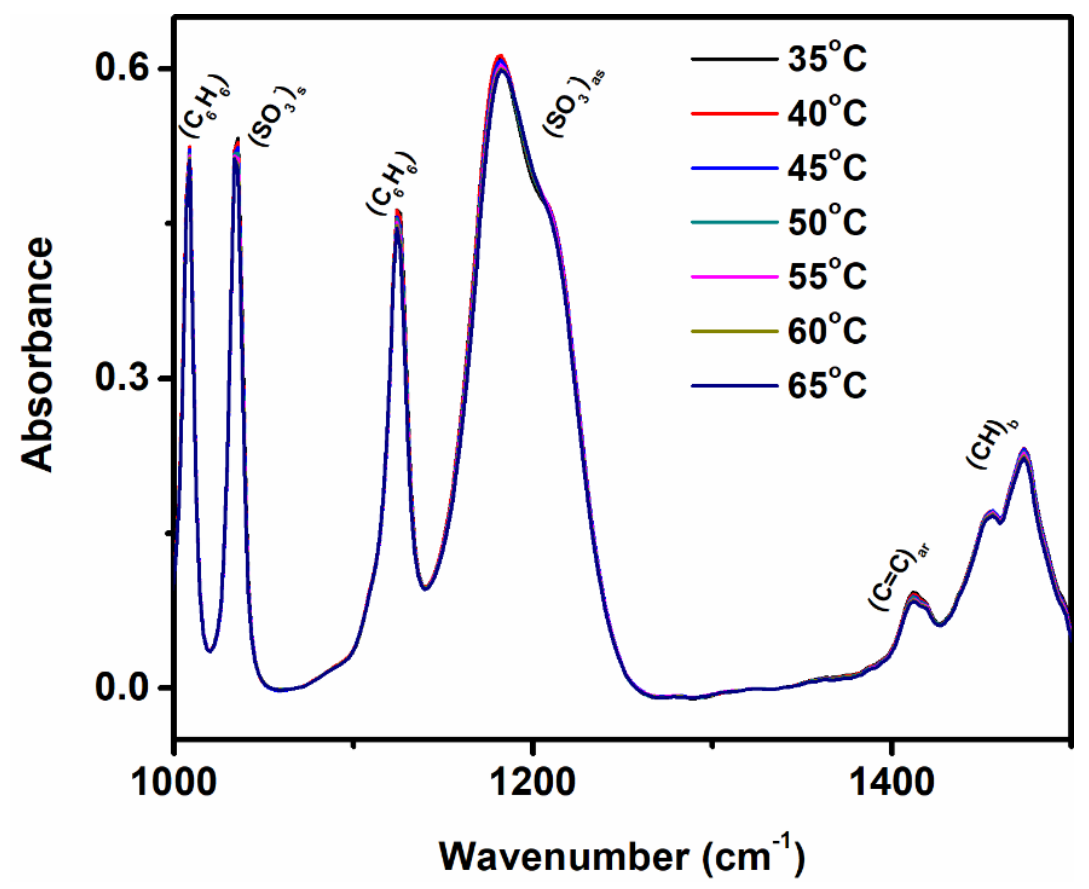

Figure 3.5: Set of baselined FTIR spectra of the polymer fingerprint region (1000 - 1500 $\mathrm{cm}^{-1}$ ) of a 70-layer pair PDADMA/PSS film assembled in $0.50 \mathrm{M} \mathrm{NaCl}$ solution and immersed in $0.50 \mathrm{M} \mathrm{NaCl} \mathrm{HOD}$ solution at temperatures from $35-65{ }^{\circ} \mathrm{C}$

A zoomed-in image of the fingerprint region is shown in Figure 3.5 detailing the characteristic peaks at 1007, 1034, 1124, 1191, 1419 and $1472 \mathrm{~cm}^{-1}$. The peak at $1472 \mathrm{~cm}^{-}$ ${ }^{1}$ is assigned to the contribution due to bending of the C-H bond from PDADMA and the peak at $1419 \mathrm{~cm}^{-1}$ is assigned to the $\mathrm{C}=\mathrm{C}$ rocking contribution of PSS. ${ }^{73}$ Symmetric and anti-symmetric vibrations of the $\mathrm{SO}_{3}{ }^{-}$group appear as the 1034 and $1191 \mathrm{~cm}^{-1}$ peaks, respectively. ${ }^{36,73,91}$ Peaks at 1007 and $1124 \mathrm{~cm}^{-1}$ are assigned to the in-plane vibration and in-plane bending vibration of benzene ring. ${ }^{91,92}$ 


\subsection{Effects of temperature on PDADMA/PSS PEMs}

Figure 3.5 shows that there was no change in the fingerprint region spectra with increasing temperature. This result enables the deduction that the amount of polyelectrolyte molecules present in the multilayer is not affected by the temperature variations.

Figures $3.6 \mathrm{a}$ and $\mathrm{b}$, which show in detail the $\mathrm{OH}$ and $\mathrm{OD}$ stretching peaks, reveal a decrease in peak area as temperature increases up to $70{ }^{\circ} \mathrm{C}$, indicating a reduced amount of water present in the films. Also, as temperature increased, a shift in the peak position from $2524 \mathrm{~cm}^{-1}$ to $2535 \mathrm{~cm}^{-1}$ was observed in the OD stretch peak shown in Figure 3.6b. This peak shift to higher wavenumber is indicative of a decrease in hydrogen bonding amongst the water molecules as temperature increases and a change to more tightly bound

water. ${ }^{86}$ For example, water in the form of ice has its OD stretch peak center at $2440 \mathrm{~cm}^{-1}$ but in the form of water vapor, the peak center is at $2719 \mathrm{~cm}^{-1} .{ }^{93}$ The data presented in both Figures $3.6 \mathrm{a}$ and $\mathrm{b}$ reflect the qualitative shift of water populations to higher frequencies and also that water uptake into the multilayers is reduced with temperature.

All results from Figure 3.5 and 3.6 combined reveal that while polymer chain mobility and conformation may change, the polymer content present in PDADMA/PSS PEMs remain the same upon thermal changes. On the other, both water content and water binding energy are largely influenced by temperature. 

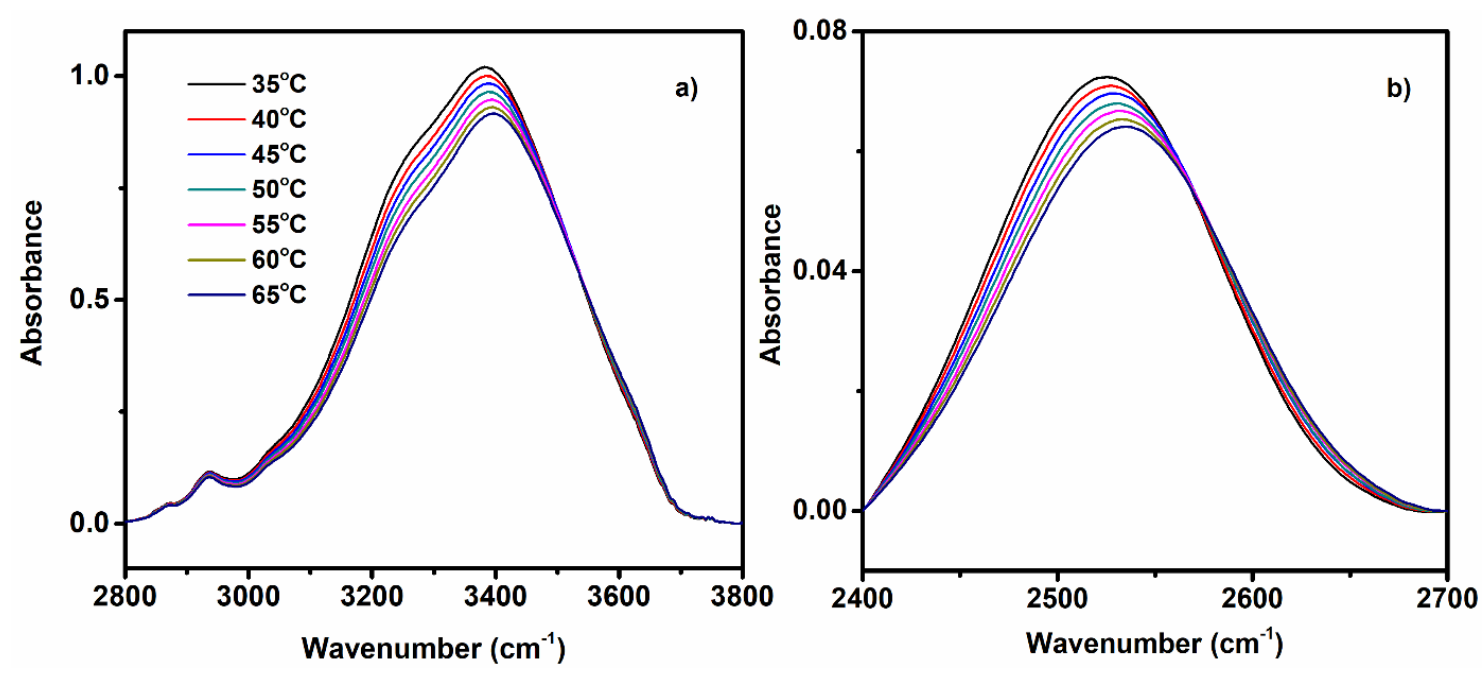

Figure 3.6: Set of baselined FTIR spectra of a) the OH stretching region (2800 - $3800 \mathrm{~cm}^{-}$

$\left.{ }^{1}\right)$, b) the OD stretching region $\left(2400-2700 \mathrm{~cm}^{-1}\right)$ of a 70-layer pair PDADMA/PSS film assembled in $0.50 \mathrm{M} \mathrm{NaCl}$ solution and immersed in $0.50 \mathrm{M} \mathrm{NaCl} \mathrm{HOD}$ solution at temperatures from $35-65^{\circ} \mathrm{C}$

The deconvolution of the OD stretch peak of the multilayer immersed in varying concentrations $(0.25-1.5 \mathrm{M})$ of $\mathrm{NaCl} \mathrm{HOD}$ solution at $35-70{ }^{\circ} \mathrm{C}$ is shown in Figure 3.7 and Table 3.1. Previous work suggests that the peak positions of the high and low frequency peaks change with properties of the polymer, but the bulk water characteristics stay constant. ${ }^{88}$ However, only two peaks are visible in all panels in Figure 3.7, attributed to low and high frequency water; the absence of a bulk water peak indicates that there is little or no bulk water present in the PEMs. This lack of bulk water may be due to the low porosity within the PEMs, which suggests that water molecules are always within the influence of the polyelectrolyte ionic groups. 

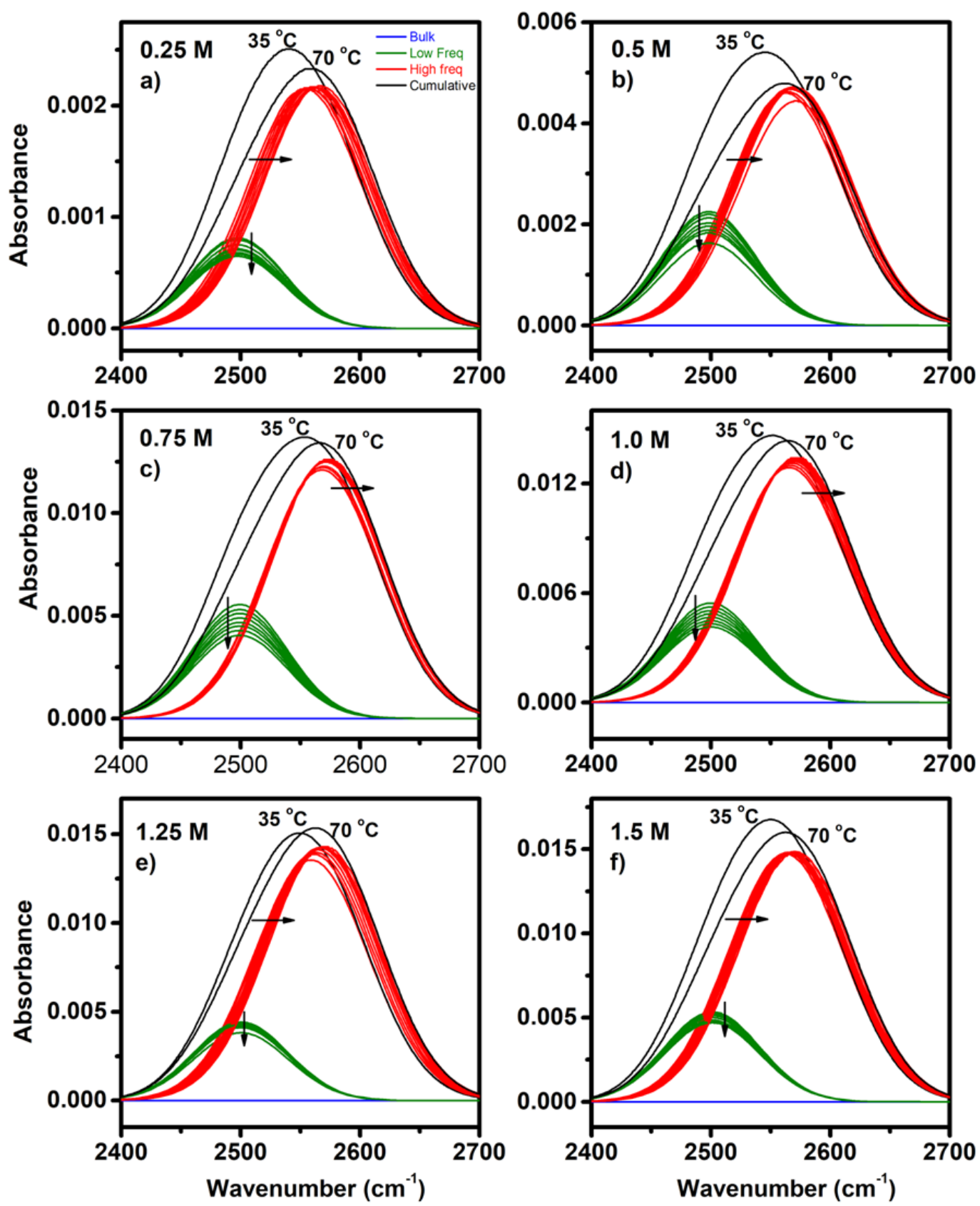

Figure 3.7: Combined deconvolutions for a PEM immersed in HOD solutions of different salt concentrations with arrows indicating the change in peak position as temperature increases from $35^{\circ} \mathrm{C}$ to $70{ }^{\circ} \mathrm{C}$. a) $0.25 \mathrm{M}$, b) $0.50 \mathrm{M}$, c) $0.75 \mathrm{M}$, d) $1.00 \mathrm{M}$, e) $1.25 \mathrm{M}$, and f) 1.50 M. Legend in (f) applies to all panels. 
Likewise, a study using modulated differential scanning calorimetry (MDSC) showed the absence of bulk water in PDADMA/PSS PECs at hydration levels of 18 to 30 $\mathrm{wt} \% .^{75}$ This indicates that the water content in the PEC either existed totally as nonfreezing bound water or with a small fraction of freezing bound, water which was identified at $30 \%$ hydration. ${ }^{75}$ Consistent with these findings, the absence of a bulk water peak from a deconvoluted OD peak was attributed to the high density of the ionic groups for homopolymer sulfonated poly(styrene).$^{88}$

Figure 3.7a shows that as the temperature increases the high frequency water peak center shifts from 2553 to $2566 \mathrm{~cm}^{-1}\left(15 \mathrm{~cm}^{-1}\right.$ shift toward effectively more tightly bound water), but a nearly constant peak area is maintained (the high frequency peak area increases by only $1.0 \%$ with increasing temperature). The lower frequency loosely bound water peak maintains its center at $2497 \pm 0.01 \mathrm{~cm}^{-1}$, while the peak area decreases by 20 $\%$. These peak shifts are consistent across all salt concentration.

In general, the FTIR spectral peak position shifts and area changes with temperature can be directly explained via increasing thermal energy of water molecules in the system. Especially for the strongly bound water molecules, the translational and rotational motion is restricted, which results in increased vibrational motion. ${ }^{75}$ The thermal motions at higher temperatures also lead to effectively weaker hydrogen bonding by the water molecules. ${ }^{93}$ However, in our data the tightly bound high frequency water peak shifts towards higher wavenumber which suggests that the PE-water associations are getting effectively stronger (hydrogen bonding strength decreases) with increasing 
temperature. This may seem counterintuitive, considering the fact that temperature, in general, should presumptively weaken the hydrogen bonds between PE and water. ${ }^{94}$

However, the reduction in cumulative water peak area means that the system is losing water which leads to a decrease in the overall electrostatic screening of water and consequently effectively stronger bonding to the material for the tightly bound water molecules.

Table 3.1: Peak information for all temperatures at $0.25 \mathrm{M} \mathrm{NaCl}$

\section{$0.25 \mathrm{M}$}

\begin{tabular}{|c|c|c|c|c|c|c|c|c|c|c|}
\hline \multicolumn{4}{|c|}{ Bulk } & \multicolumn{3}{|c|}{ Low frequency } & \multicolumn{3}{|c|}{ High frequency } & \multirow{2}{*}{$\begin{array}{l}\text { Cumulat } \\
\text { ive water } \\
\text { Area }\end{array}$} \\
\hline Temp & Area & fwhm & $\begin{array}{l}\text { Peak } \\
\text { center }\end{array}$ & Area & fwhm & $\begin{array}{l}\text { Peak } \\
\text { center }\end{array}$ & Area & fwhm & $\begin{array}{l}\text { Peak } \\
\text { center }\end{array}$ & \\
\hline 35 & $1 \mathrm{E}-9$ & 170 & 2509 & 31.5 & 92.0 & 2497.1 & 100.9 & 110.6 & 2553.7 & 132.4 \\
\hline 40 & $1 \mathrm{E}-9$ & 170 & 2509 & 30.8 & 92.0 & 2497.1 & 101.2 & 110.5 & 2556.1 & 132.0 \\
\hline 45 & $1 \mathrm{E}-9$ & 170 & 2509 & 29.1 & 92.0 & 2497.1 & 100.8 & 110.6 & 2557.4 & 129.9 \\
\hline 50 & $1 \mathrm{E}-9$ & 170 & 2509 & 27.7 & 92.0 & 2497.1 & 101.2 & 110.4 & 2559.4 & 128.9 \\
\hline 55 & $1 E-9$ & 170 & 2509 & 27.3 & 92.0 & 2497.1 & 101.5 & 110.5 & 2561.3 & 128.8 \\
\hline 60 & $1 \mathrm{E}-9$ & 170 & 2509 & 26.39 & 91.96 & 2497.1 & 101.2 & 110.4 & 2562.8 & 127.6 \\
\hline 65 & $1 \mathrm{E}-9$ & 170 & 2509 & 25.84 & 91.96 & 2497.1 & 101.2 & 110.6 & 2564.5 & 127.1 \\
\hline 70 & $1 \mathrm{E}-9$ & 170 & 2509 & 25.14 & 92.00 & 2497.1 & 102.0 & 110.4 & 2566.5 & 127.1 \\
\hline
\end{tabular}


Throughout the examined temperature range, the high frequency water peak maintained a constant peak area in Figure 3.7. This data indicates that a constant number of water molecules constitutes the high frequency water at all temperatures in the system. We suggest that all ion pairs are fully surrounded by high frequency water at all examined conditions with no change in hydration as temperature is varied. This potentially suggests that at extremely low hydrations all added water may exist as high frequency water, tightly bound to the ionic groups and hydrating them. Once the maximum amount of high frequency water is absorbed into the PEM, additional water becomes low frequency, loosely bound water and eventually bulk water. An earlier simulation work shows the saturation response of tightly bound water upon increasing hydration in partially hydrated PDADMA/PSS complexes. ${ }^{75,94}$

However, contrary to the tightly bound high frequency water, the weakly bound low frequency water exists with a fixed effective hydrogen bonding energy: its peak center remained constant at $2497 \pm 0.01 \mathrm{~cm}^{-1}$, while the peak area decreased with increasing temperature. The decrease of the low frequency peak area with temperature suggests that the absolute amount of weakly bound water molecules decreases. At the microscopic level, this is very likely related with breaking some of the weaker hydrogen bonds in the outer hydration shell, which leads to release of water molecules, perhaps into the external contacting solution. At the macroscopic level, this would indicate that, as temperature increases, the PEMs hold less water.

The results presented in Figure 3.7 can be used to complement the interpretation of previous MD simulation results on partially hydrated PDADMA-PSS PECs ${ }^{75}$ to provide 
some additional insight to the behavior of water molecules in this system. As the FTIR spectral characterization of this work was done for fully hydrated and immersed systems, we restrict the consideration to the temperature response. Both the data in Figure 3.7 and previous reports ${ }^{75}$ suggest that most of the water in the PEM is strongly bound even at high water content. Figure 3.7, moreover, indicates that strongly bound water dominates also in fully hydrated PDADMAC/PSS systems.

\subsection{Effects of ionic strength on PDADMA/PSS PEMs}

In general, the uptake of water swells the PEM; specifically, a 4-layer pair of PDADMA/PSS capped with PDADMA prepared in Schlenoff and Dubas showed that PDADMA/PSS films prepared in the presence of $1.0 \mathrm{M} \mathrm{NaCl}$ swell up to 2.03 times that of the dry thickness when immersed in pure water. ${ }^{64}$ However, the films had a lower swelling ratio when immersed in $<0.7 \mathrm{M} \mathrm{NaCl}$ solutions, and higher ratios when immersed in $>0.7 \mathrm{M} \mathrm{NaCl}^{64}$ Similarly, a decrease in thickness or no change was observed in PDADMA/PSS films (assembled from $0.5 \mathrm{M} \mathrm{NaCl})$ when exposed to $0.1 \mathrm{M}(\sim 5 \%$ thickness decrease) and $0.5 \mathrm{M}$ ( $\sim 0 \%$ thickness change) $\mathrm{KBr}$ post assembly whereas films exposed to $\mathrm{KBr}$ solutions of lower or higher concentrations experienced swelling. ${ }^{45}$ These works show that PDADMA/PSS PEMs may swell or slightly contract depending on the doping salt concentration, the capping layer, and assembly conditions. 


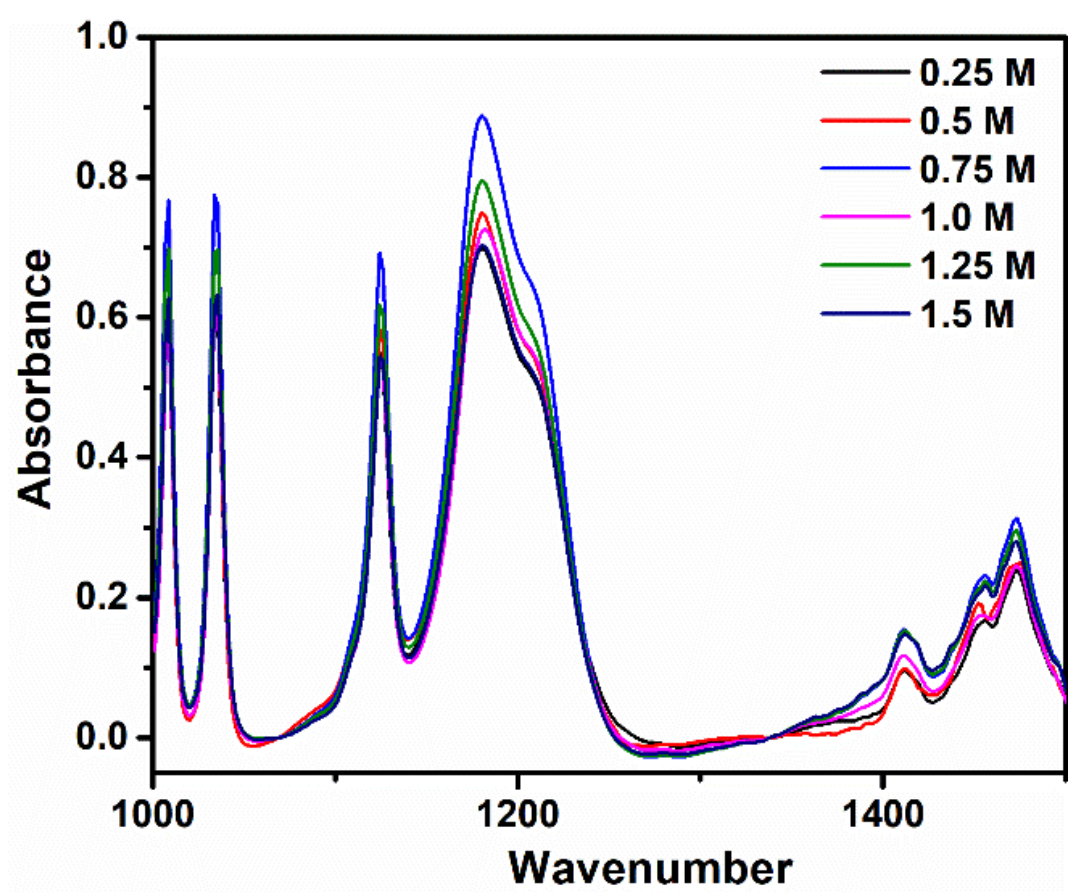

Figure 3.8: FTIR spectra of fingerprint region at varying salt concentrations

However, the introduction of counterions into the PEMs post-assembly causes variations in the sulfonate anti-symmetric stretch peak (Figures 3.8 and 3.9). In brief, the sulfonate anti-symmetric peak increases in area with the addition of salt up to $0.75 \mathrm{M}$ $\mathrm{NaCl}$, and then decreases in area with higher ionic strengths. This is attributed to polyelectrolyte and anti-polyelectrolyte effects, ${ }^{95}$ as well as the increased formation of extrinsic ion pairs via screening effects. ${ }^{45}$ An increase in peak area is observed as $\mathrm{NaCl}$ concentration increased to $0.75 \mathrm{M}$. This can be explained by an increase in PE-counterion pairing upon addition of salt, leading to tighter packed assemblies. Above $0.75 \mathrm{M}$, the PEMs experience swelling due to electrostatic screening effects leading to a drop in peak area. 


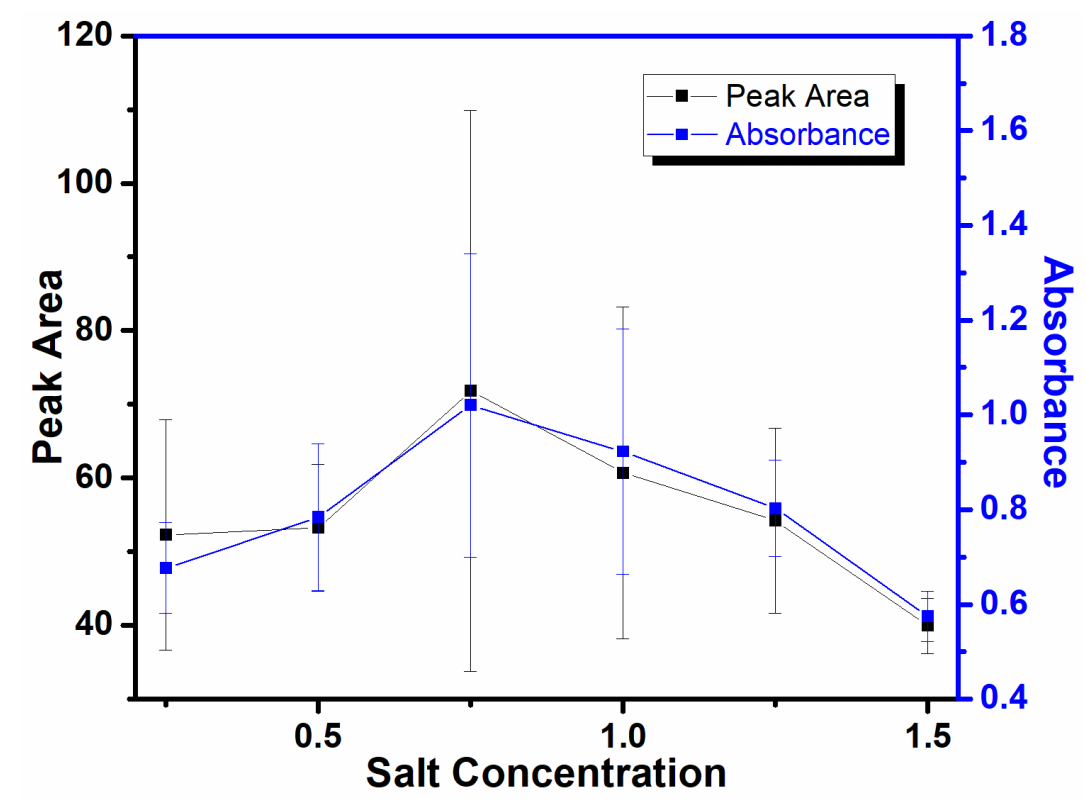

Figure 3.9: A relationship between the $\mathrm{SO}_{3}{ }^{-}$anti-symmetric stretch peak (at $1191 \mathrm{~cm}^{-1}$ ) and salt concentration obtained from Figure 3.8

Figure 3.10 compares the deconvolution of OD stretch peaks for varying salt concentrations of $\mathrm{NaCl}$ in $\mathrm{HOD}$ at a constant temperature of $40{ }^{\circ} \mathrm{C}$. While the peak area of all the cumulative $\mathrm{OD}$ stretch peaks generally increased with increasing $\mathrm{NaCl}$ concentration, the cumulative peak center at all concentrations remained approximately constant. The deconvolution of the cumulative OD peak also shows that the areas of the high and low frequency water peaks increased with the addition of salt (Figures $3.10 \mathrm{c}$ and d). These results indicate the PEMs swell with water as the ionic strength of the contacting solution increases. 

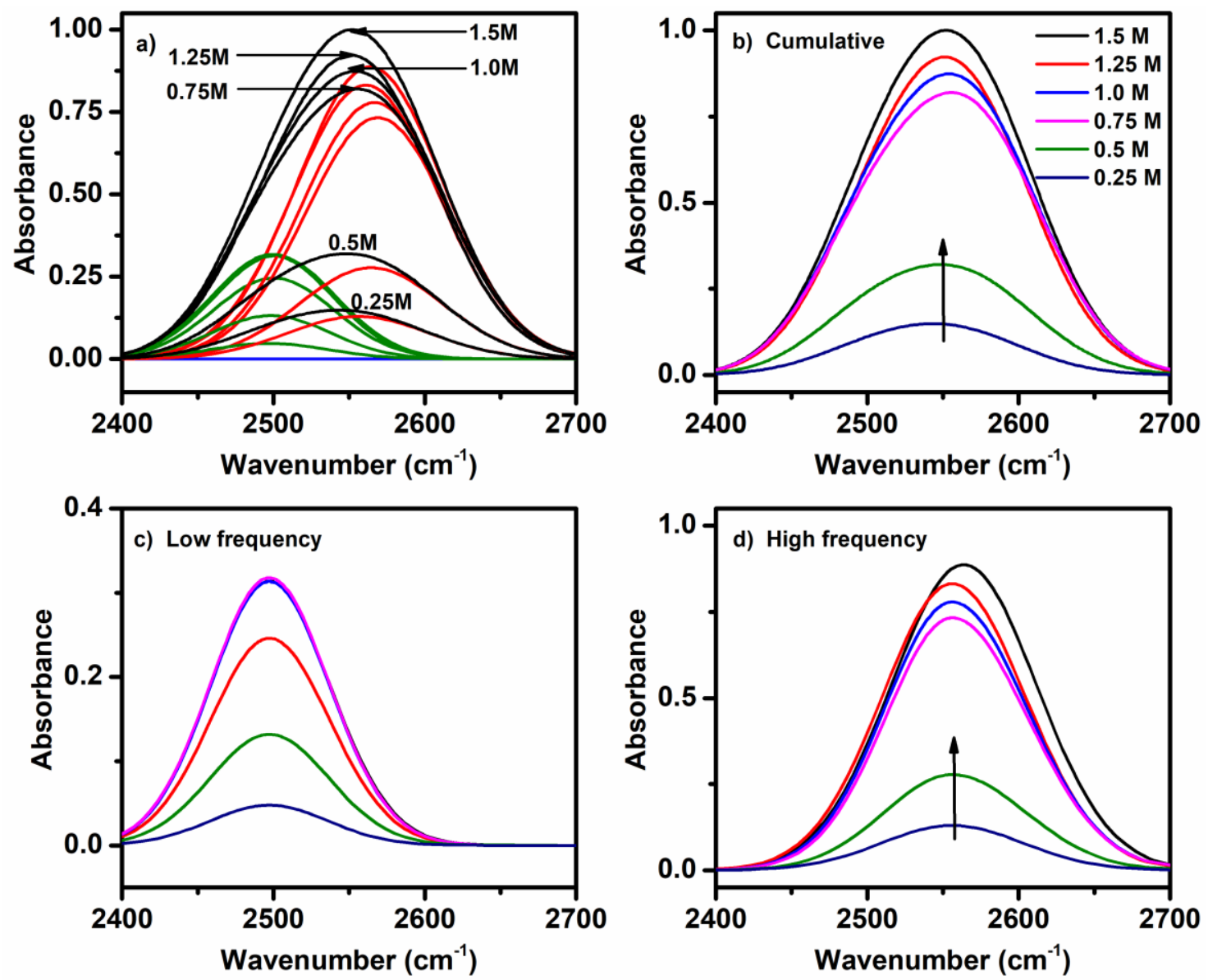

Figure 3.10: a) Combined deconvolutions for a PEM immersed in HOD solutions of different salt concentrations at $40{ }^{\circ} \mathrm{C}$ showing the cumulative (black), high (red) and low frequency water (green), and bulk water (blue) peaks. b) Cumulative c) Low frequency d) High frequency water peaks at different salt concentrations at $40{ }^{\circ} \mathrm{C}$ obtained from Figure 3.10a for clarity. The arrows in (b) and (d) indicate the trend with increasing salt concentration

This finding is in accordance with previous studies of PDADMA/PSS multilayers in the presence of $\mathrm{KBr}$ using QCM-D, ${ }^{45}$ where the swelling response showed different regions of response as a function of the salt concentration of the contacting solution. 
The origin of this behavior has two features: an increase in ionic strength 1) breaks intrinsic polycation-polyanion pairs, allowing for an increase in free volume of the PEM network and 2) presumably causes swelling due to incoming water molecules that are hydrating the absorbed salt ions. This can be expected to lead to varying degrees of swelling of the multilayer, and previous simulation works also show that the presence of ions influences the water structure and PE-PE binding. ${ }^{58,96,97}$. In addition to examining the changes in peak area, we also sought to inspect changes in peak center; however, the peak center shifts were very subtle and within the resolution of the instrument $\left(\sim 4 \mathrm{~cm}^{-1}\right)$.

A possible explanation for these variations may be related to the dual nature of salt in polyelectrolyte assemblies. ${ }^{58}$ Salt ions act as plasticizer to the PEMs by bringing additional water molecules, which is clearly demonstrated by the increasing peak area in Figure 3.10a. On the other hand, salt ions strongly bind water molecules through hydration of the ions and, therefore, compete with the polyelectrolytes for water interactions. At higher salt concentration, this effect can be pronounced enough to influence the hydration shell around polyelectrolytes by altering the number of water molecules or by altering the polyelectrolyte-water binding strength. As suggested by molecular dynamics simulations for the PDADMAC/PSS system, ${ }^{58}$ the increase in salt concentration decreases the amount of water around PSS and simultaneously increases the PSS-water hydrogen bond strength (or hydrogen bond lifetime). The increase in binding strength, as mentioned above, may be manifested by the shift in the high frequency peak center towards higher values. As can be seen in Table 3.2, at a constant temperature, a slight shift in high frequency peak center with increasing salt concentration can be observed. Altogether, our results combined with 
previous considerations, ${ }^{45,58}$ suggest that salt is changing the nature of polyelectrolytewater binding.

Table 3.2: Peak information for all salt concentrations at $40{ }^{\circ} \mathrm{C}$

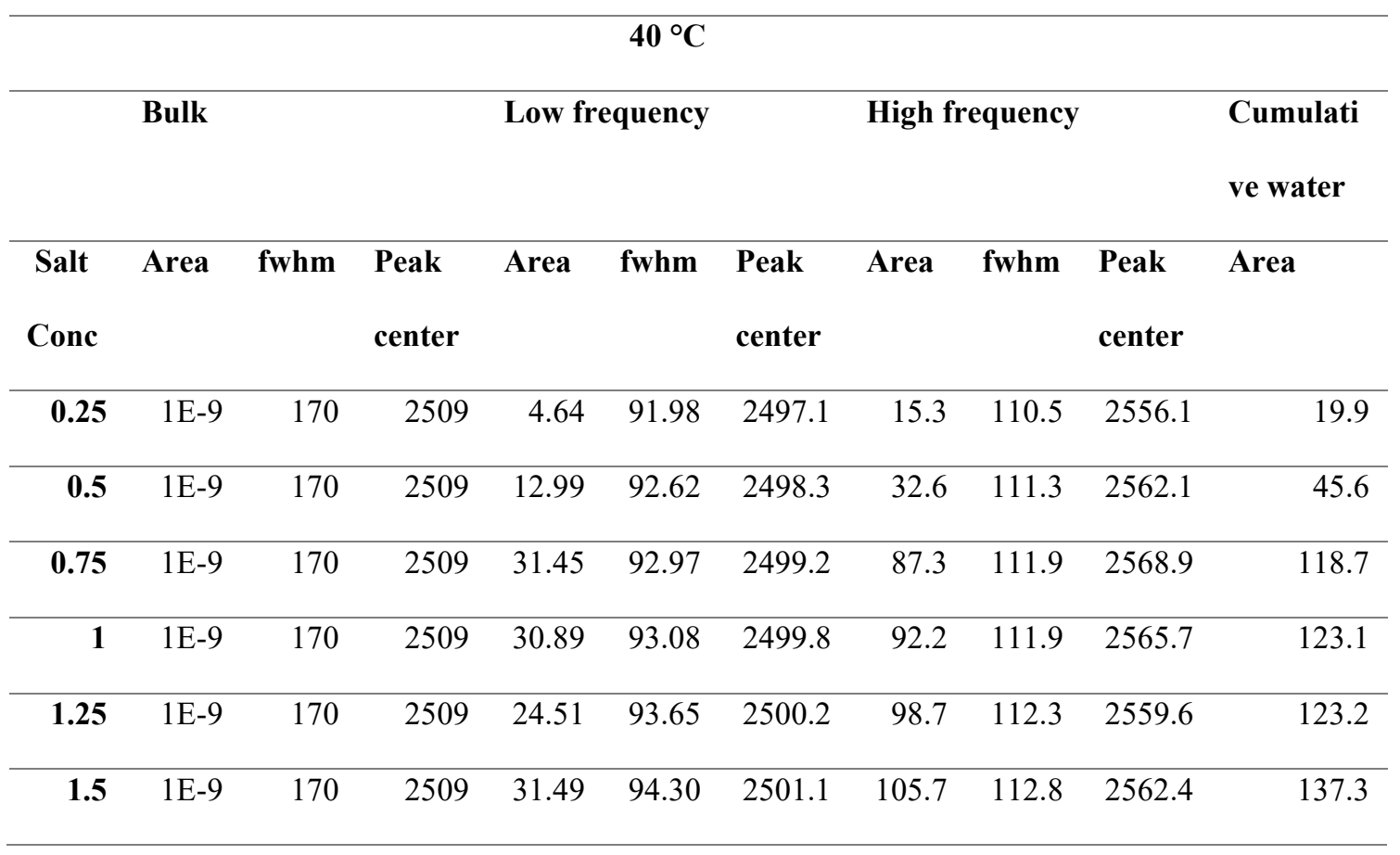




\subsection{Combined effects of temperature and ionic strength on PEMs}
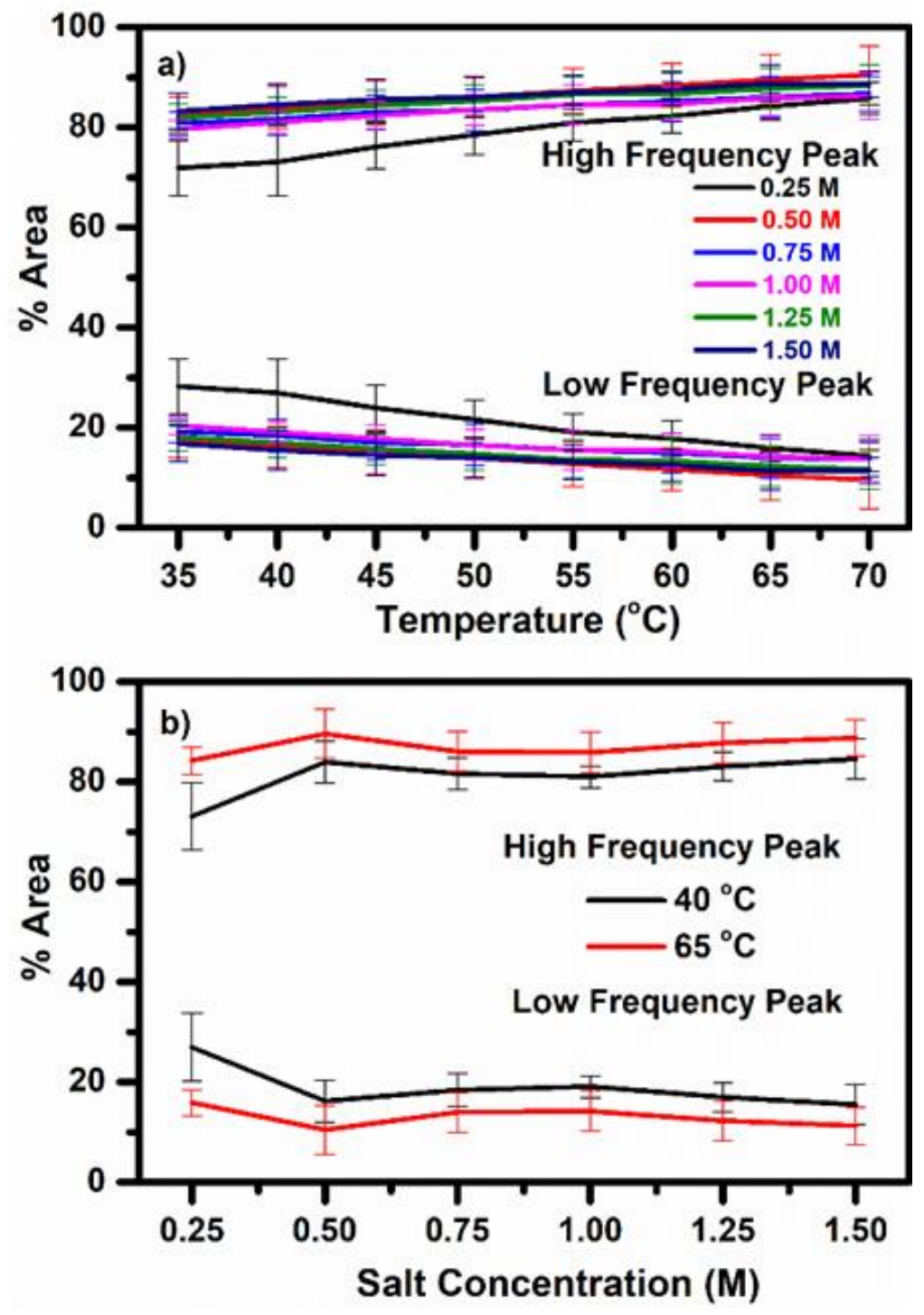

Figure 3.11: (a) Population distribution of water as percent area of each state for all tested salt concentrations and temperatures. (b) Comparison of percent area of low and high frequency peaks with respect to increasing salt concentration at $40{ }^{\circ} \mathrm{C}$ (black) and $60{ }^{\circ} \mathrm{C}$ (red). The two top-most (higher percentage) lines correspond to the high frequency water peaks and the two bottom-most (lower percentage) correspond to the low frequency water peaks. 
Figure 3.11 demonstrates a comparison of the effects of temperature and salt concentration on the population distribution of water within the PDAMA/PSS PEM. Figure 3.11a shows the variation of high frequency and low frequency OD peak areas with temperature at different $\mathrm{NaCl}$ concentrations. The results show that about $80 \%$ of water molecules contribute to the high-frequency peak and are more tightly bound; also, the relative peak area percentages do not change much with regard to temperature. Figure $3.11 \mathrm{~b}$ compares the effects of salt concentration for two different temperatures, 40 and $65{ }^{\circ} \mathrm{C}$, on the population distribution of water within the PDADMA/PSS PEM. Similarly, there is little variation in the relative contributions of the high and low frequency peaks with regard to salt concentration. At $40{ }^{\circ} \mathrm{C}$, the relative contribution of high frequency water is slightly reduced, but remains within error of the contribution at $65^{\circ} \mathrm{C}$.

\subsection{Van't Hoff relationship}

To obtain more insight into the temperature response of water within the PEMs, a van't Hoff plot was constructed, Figure 3.12. The plot is based on the high and low frequency water populations from the deconvolution of OD stretch peaks and shows the natural logarithm of the ratio of the high frequency to low frequency water population against the inverse of temperature for each salt concentration of PEM. This analysis considers the equilibrium "reaction" of low frequency water shifting its state to high

frequency water, equation 3.1. Similar works ${ }^{98,} 99$ have been done based on the same assumption that the water $\mathrm{OH}$ spectra can be deconvoluted into two water components: 

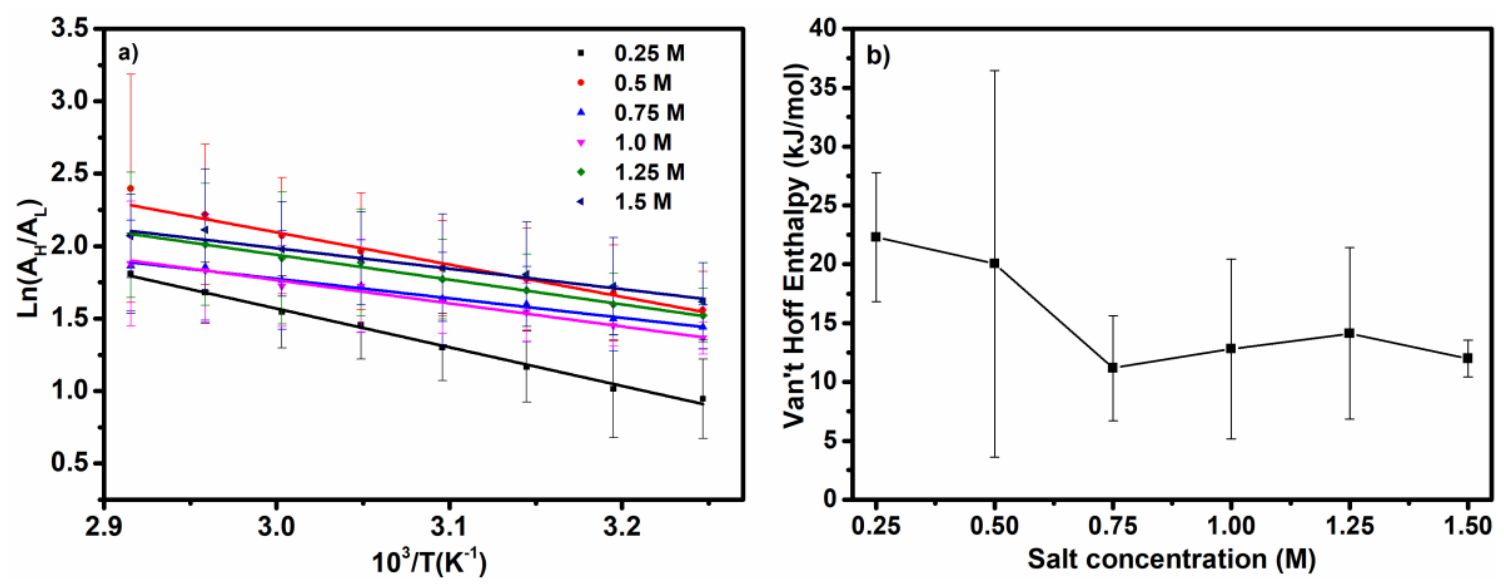

Figure 3.12: van't Hoff plot produced using the percent areas from Figure 3.11. The lines represent linear fits using the van’t Hoff relationship. b) van't Hoff enthalpy dependence on salt concentration

correlated (intermediate) water and non-correlated (free) water. An equilibrium isosbestic point, identified by equal absorption, is determined as a point of division for the two water components. Therefore, by assuming that the equilibrium of the reaction may be represented as the ratio of the high frequency water concentration to the low frequency water concentration, we can represent the van't Hoff equation as equation 3.2.

Furthermore, by assuming that each water population is proportional to its respective FTIR peak area, the van't Hoff equation may be modified to equation 3.3. This relationship predicts that the slope and y-intercept of the van't Hoff plot will yield the enthalpy and entropy of the conversion of low frequency water to high frequency water. ${ }^{99}$

$$
\mathrm{H}_{2} \mathrm{O} \mathrm{I}_{L} \leftrightarrow \mathrm{H}_{2} \mathrm{O} \mathrm{I}_{H}
$$

equation 3.1 


$$
\begin{gathered}
K_{e q}=\frac{\left[\left.\mathrm{H}_{2} O\right|_{H}\right]}{\left[\left.\mathrm{H}_{2} O\right|_{L}\right]}=\frac{A_{H}}{A_{L}} \\
\ln K_{e q}=\ln \frac{A_{H}}{A_{L}}=-\frac{\Delta H}{R T}+\frac{\Delta S}{R}
\end{gathered}
$$

equation 3.3

In accordance with typical van't Hoff behavior, the data presents a linear trend of $\ln \left(A_{H} / A_{L}\right) \sim 1 / T$. The linear trend suggests that the enthalpy and entropy are independent of temperature for the experiment. ${ }^{98}$ However, the differences in the slope as salt varies indicates a salt-dependence of the van't Hoff enthalpy relationship. Notably in Figure 3.12a, the data sets corresponding to $0.25 \mathrm{M}$ and $0.5 \mathrm{M}$ have the greatest slopes. This means that, for these data sets, the ratio of the high frequency peak area to low frequency peak area is most sensitive to temperature change. In practice, the peak area change occurs mostly for the low frequency water peak but not so much for the high frequency peak. This means that these intermediate salt concentrations are more sensitive to the release of weakly bound water with increasing temperature. Figure $3.12 \mathrm{~b}$, shows that the calculated van't Hoff enthalpy values range from $11-22 \mathrm{~kJ} / \mathrm{mol}(2.6-5.2 \mathrm{kcal} / \mathrm{mol})$ across the different salt concentrations studied. Similarly, the van't Hoff entropy values range from $48-79 \mathrm{~kJ} / \mathrm{molK}(11.5-19 \mathrm{kcal} / \mathrm{molK})$. Using various techniques including Raman and IR spectroscopy, light and neutron scattering, and molecular dynamics, the van't Hoff enthalpy of liquid water has been obtained within the range from $2.3-3 \mathrm{kcal} / \mathrm{mol},{ }^{100-102}$ which is of similar value here. This van't Hoff enthalpy represents the breakage of a hydrogen bond across the two water microenvironments present in the PEM system. ${ }^{101}$ 
The changes in van't Hoff enthalpy with ionic strength may be as a result of the screening effects of the salt ions present around water molecules. Also, results from MD simulations by Batys et $a l .{ }^{75}$ may be employed to shed more light on the endothermic nature of the van't Hoff enthalpy. The increase in rotational diffusion of water molecules within PDADMA/PSS PECs with increasing temperature ${ }^{103104103103103104104104104104104104104}$ might suggest that the vibrational frequency of water molecules becomes faster as temperature increases. There is therefore a transition from slower to faster vibrations with temperature increase. 


\section{CHAPTER IV}

\section{CONCLUSIONS AND FUTURE WORK}

\subsection{Conclusions}

The influence of salt concentration and temperature on the hydrogen bonding of water molecules (specifically, HOD molecules) within PDADMA/PSS films was studied via ATR-FTIR spectroscopy. Deconvolution of OD stretch peak led to the conclusion that most of the water molecules in a fully hydrated PEM are located within regions of influence with the polymers (tightly or loosely bound), regardless of salt concentration or temperature. An increase in temperature caused an overall decrease in the amount of water within the film, but the amount of tightly bound water remained constant, indicating that water was released only from the outer hydration shells of the ionic groups. Increasing the salt concentration led to the disruption of intrinsic polycation-polyanion pairs, which led to swelling of the film, as evidenced by the relative increase in the OD stretch peak area. The high and low frequency peak areas were quantified, and the peak area ratio followed a van't Hoff-type relationship. The van't Hoff enthalpy was in the range of $11-22 \mathrm{~kJ} / \mathrm{mol}$, which is in accordance with a typical hydrogen bonding response.

Altogether in this study, we showed that the water content in fully hydrated PEMs is influenced both by salt concentration and temperature, while the binding and interactions nature of individual polyelectrolyte molecules in the multilayers are controlled mostly by the salt counterions. Temperature plays a role via thermal vibrations, but it was not observed to change the binding character of the polyelectrolytes. An 
assessment of the distribution of water binding around the ion pairs was also made.

Microenvironments of water molecules with high and low effective hydrogen bonding energy were identified; the amount of high frequency water remained relatively constant with temperature and salt indicating consistent hydration of the tightly bound water species in the PEM; meanwhile, the amount of low frequency water decreased with temperature indicating a loss of some of the more weakly hydrogen bonded water molecules with increasing temperature. The results provide insight into the character of water in PEMs but also lay foundation to understanding the varied microenvironments of water molecules within any charged system, whether it be synthetic or biological.

\subsection{Future Work}

Following the results obtained from this research, future work would involve conducting similar studies using ATR-FTIR spectroscopy on weak PAH/PAA PEMs to determine response to both temperature and $\mathrm{pH}$. Although this technique enables us identify water microenvironments by virtue of their binding energy to all polyelectrolyte ion pairs, it is also important to identify the particular ion pair water molecules associate with. That is, identifying the number of water molecules associated with intrinsic ion pairs and each kind of extrinsic ion pair. This is important as Zhang et al. have proved for both strong and weak polyelectrolyte systems, that the glass transition temperature of both

PEMs and PECs can be related to the ratio of water molecules to intrinsic ion pairs. ${ }^{44}$ One possible characterization technique with this potential is quartz crystal microbalance (QCM). QCM operates on the piezo-electricity of a quartz crystal sandwich between two 
electrodes. By so doing, the water content of the PEMs can be determined from the mass and thickness of PEMs at both hydrated and dry states. 


\section{REFERENCES}

1. de Jong, B., Coacervation. Proc. Royal Acad. Amsterdam 1929, 32, 849-856.

2. Parveen, N.; Schönhoff, M., Swelling and Stability of Polyelectrolyte Multilayers in Ionic Liquid Solutions. Macromolecules 2013, 46 (19), 7880-7888.

3. Holder, K. M.; Smith, R. J.; Grunlan, J. C., A review of flame retardant nanocoatings prepared using layer-by-layer assembly of polyelectrolytes. Journal of Materials Science 2017, 52 (22), 12923-12959.

4. Suarez-Martinez, P. C.; Robinson, J.; An, H.; Nahas, R. C.; Cinoman, D.; Lutkenhaus, J. L., Polymer-clay nanocomposite coatings as efficient, environmentfriendly surface pretreatments for aluminum alloy 2024-T3. Electrochimica Acta 2018, $260,73-81$.

5. Ahmadiannamini, P.; L. Bruening, M.; Tarabara, V., Sacrificial polyelectrolyte multilayer coatings as an approach to membrane fouling control: Disassembly and regeneration mechanisms. Journal of Membrane Science 2015, 491.

6. Min, J.; Braatz, R. D.; Hammond, P. T., Tunable staged release of therapeutics from layer-by-layer coatings with clay interlayer barrier. Biomaterials 2014, 35 (8), 2507 2517.

7. Sill, A.; Nestler, P.; Azinfar, A.; Helm, C. A., Tailorable Polyanion Diffusion Coefficient in LbL Films: The Role of Polycation Molecular Weight and Polymer Conformation. Macromolecules 2019, 52 (22), 9045-9052. 
8. Morawetz, H., Polyelectrolytes, science and technology, Hara Masanori, ed., Marcel Dekker, New York, 1993, 416 pp. price: \$150.00. Journal of Polymer Science Part A: Polymer Chemistry 1993, 31 (9), 2413-2413.

9. Dobrynin, A. V.; Rubinstein, M., Hydrophobic Polyelectrolytes. Macromolecules 1999, 32 (3), 915-922.

10. Dobrynin, A. V.; Rubinstein, M., Theory of polyelectrolytes in solutions and at surfaces. Progress in Polymer Science 2005, 30 (11), 1049-1118.

11. Zhu, T.; Sha, Y.; Yan, J.; Pageni, P.; Rahman, M. A.; Yan, Y.; Tang, C., Metallopolyelectrolytes as a class of ionic macromolecules for functional materials. Nature Communications 2018, 9 (1), 4329.

12. Kawamura, S., Effectiveness of Natural Polyelectrolytes in Water Treatment. Journal (American Water Works Association) 1991, 83 (10), 88-91.

13. Wang, Y.; Shen, Y.; Zhang, Y.; Yue, B.; Wu, C., pH-Sensitive Polyacrylic Acid (PAA) Hydrogels Trapped with Polysodium-p-Styrenesulfonate (PSS). Journal of Macromolecular Science, Part B 2006, 45 (4), 563-571.

14. Rathee, S. V.; Sidky, H.; Sikora, J. B.; Whitmer, K. J., Explicit Ion Effects on the Charge and Conformation of Weak Polyelectrolytes. Polymers 2019, 11 (1).

15. Mortimer, D. A., Synthetic polyelectrolytes-A review. Polymer International 1991, 25 (1), 29-41.

16. Löhmann, O.; Zerball, M.; von Klitzing, R., Water Uptake of Polyelectrolyte Multilayers Including Water Condensation in Voids. Langmuir 2018, 34 (38), 1151811525. 
17. Guzmán, E.; Ritacco, H.; Rubio, J. E. F.; Rubio, R. G.; Ortega, F., Salt-induced changes in the growth of polyelectrolyte layers of poly(diallyl-dimethylammonium chloride) and poly(4-styrene sulfonate of sodium). Soft Matter 2009, 5 (10), 2130-2142.

18. Chollakup, R.; Smitthipong, W.; Eisenbach, C. D.; Tirrell, M., Phase Behavior and Coacervation of Aqueous Poly(acrylic acid)-Poly(allylamine) Solutions. Macromolecules 2010, 43 (5), 2518-2528.

19. Ali, S.; Prabhu, V. M., Relaxation Behavior by Time-Salt and Time-Temperature Superpositions of Polyelectrolyte Complexes from Coacervate to Precipitate. Gels 2018, $4(1), 11$.

20. McAloney, R. A.; Dudnik, V.; Goh, M. C., Kinetics of Salt-Induced Annealing of a Polyelectrolyte Multilayer Film Morphology. Langmuir 2003, 19 (9), 3947-3952.

21. Miller, M. D.; Bruening, M. L., Correlation of the Swelling and Permeability of Polyelectrolyte Multilayer Films. Chemistry of Materials 2005, 17 (21), 5375-5381.

22. Choi, J.; Rubner, M. F., Influence of the Degree of Ionization on Weak Polyelectrolyte Multilayer Assembly. Macromolecules 2005, 38 (1), 116-124.

23. Zhang, Y.; Yildirim, E.; Antila, H. S.; Valenzuela, L. D.; Sammalkorpi, M.; Lutkenhaus, J. L., The influence of ionic strength and mixing ratio on the colloidal stability of PDAC/PSS polyelectrolyte complexes. Soft Matter 2015, 11 (37), 7392-7401.

24. Wang, Q.; Schlenoff, J. B., The Polyelectrolyte Complex/Coacervate Continuum. Macromolecules 2014, 47 (9), 3108-3116. 
25. Li, L.; Srivastava, S.; Andreev, M.; Marciel, A. B.; de Pablo, J. J.; Tirrell, M. V., Phase Behavior and Salt Partitioning in Polyelectrolyte Complex Coacervates. Macromolecules 2018, 51 (8), 2988-2995.

26. Soltwedel, O.; Nestler, P.; Neumann, H.-G.; Paßvogel, M.; Köhler, R.; Helm, C. A., Influence of Polycation (PDADMAC) Weight on Vertical Diffusion within Polyelectrolyte Multilayers during Film Formation and Postpreparation Treatment. Macromolecules 2012, 45 (19), 7995-8004.

27. Zhang, Y.; Li, F.; Valenzuela, L. D.; Sammalkorpi, M.; Lutkenhaus, J. L., Effect of Water on the Thermal Transition Observed in Poly(allylamine hydrochloride)Poly(acrylic acid) Complexes. Macromolecules 2016, 49 (19), 7563-7570.

28. Fu, J.; Abbett, R. L.; Fares, H. M.; Schlenoff, J. B., Water and the Glass Transition Temperature in a Polyelectrolyte Complex. ACS Macro Letters 2017, 6 (10), 1114-1118. 29. Shao, L.; Lutkenhaus, J. L., Thermochemical properties of free-standing electrostatic layer-by-layer assemblies containing poly(allylamine hydrochloride) and poly(acrylic acid). Soft Matter 2010, 6 (14), 3363-3369.

30. Iler, R. K., Multilayers of colloidal particles. Journal of Colloid and Interface Science 1966, 21 (6), 569-594.

31. Decher, G.; Hong, J. D.; Schmitt, J., Buildup of ultrathin multilayer films by a self-assembly process: III. Consecutively alternating adsorption of anionic and cationic polyelectrolytes on charged surfaces. Thin Solid Films 1992, 210-211, 831-835.

32. Swenson, H.; Stadie, N. P., Langmuir's Theory of Adsorption: A Centennial Review. Langmuir 2019, 35 (16), 5409-5426. 
33. Langmuir, I., THE ADSORPTION OF GASES ON PLANE SURFACES OF GLASS, MICA AND PLATINUM. Journal of the American Chemical Society 1918, 40 (9), 1361-1403.

34. Decher, G., Fuzzy Nanoassemblies: Toward Layered Polymeric Multicomposites. Science 1997, 277 (5330), 1232-1237.

35. Iturri Ramos, J. J.; Stahl, S.; Richter, R. P.; Moya, S. E., Water Content and Buildup of Poly(diallyldimethylammonium chloride)/Poly(sodium 4-styrenesulfonate) and Poly(allylamine hydrochloride)/Poly(sodium 4-styrenesulfonate) Polyelectrolyte Multilayers Studied by an in Situ Combination of a Quartz Crystal Microbalance with Dissipation Monitoring and Spectroscopic Ellipsometry. Macromolecules 2010, 43 (21), 9063-9070.

36. Farhat, T.; Yassin, G.; Dubas, S. T.; Schlenoff, J. B., Water and Ion Pairing in Polyelectrolyte Multilayers. Langmuir 1999, 15 (20), 6621-6623.

37. Jaber, J. A.; Schlenoff, J. B., Recent developments in the properties and applications of polyelectrolyte multilayers. Current Opinion in Colloid \& Interface Science 2006, 11 (6), 324-329.

38. Bieker, P.; Schönhoff, M., Linear and Exponential Growth Regimes of Multilayers of Weak Polyelectrolytes in Dependence on pH. Macromolecules 2010, 43 (11), 50525059.

39. v. Klitzing, R., Internal structure of polyelectrolyte multilayer assemblies. Physical Chemistry Chemical Physics 2006, 8 (43), 5012-5033. 
40. Li, Y.; Wang, X.; Sun, J., Layer-by-layer assembly for rapid fabrication of thick polymeric films. Chemical Society Reviews 2012, 41 (18), 5998-6009.

41. Steitz, R.; Jaeger, W.; Klitzing, R. v., Influence of Charge Density and Ionic Strength on the Multilayer Formation of Strong Polyelectrolytes. Langmuir 2001, 17 (15), 4471-4474.

42. Dubas, S. T.; Schlenoff, J. B., Polyelectrolyte Multilayers Containing a Weak Polyacid: Construction and Deconstruction. Macromolecules 2001, 34 (11), 3736-3740.

43. Kharlampieva, E.; Sukhishvili, S. A., Ionization and pH Stability of Multilayers Formed by Self-Assembly of Weak Polyelectrolytes. Langmuir 2003, 19 (4), 1235-1243. 44. Zhang, Y.; Batys, P.; O’Neal, J. T.; Li, F.; Sammalkorpi, M.; Lutkenhaus, J. L., Molecular Origin of the Glass Transition in Polyelectrolyte Assemblies. ACS Central Science 2018, 4 (5), 638-644.

45. O’Neal, J. T.; Dai, E. Y.; Zhang, Y.; Clark, K. B.; Wilcox, K. G.; George, I. M.; Ramasamy, N. E.; Enriquez, D.; Batys, P.; Sammalkorpi, M.; Lutkenhaus, J. L., QCM-D Investigation of Swelling Behavior of Layer-by-Layer Thin Films upon Exposure to Monovalent Ions. Langmuir 2018, 34 (3), 999-1009.

46. Izumrudov, V.; Kharlampieva, E.; Sukhishvili, S. A., Salt-Induced Multilayer Growth: Correlation with Phase Separation in Solution. Macromolecules 2004, 37 (22), 8400-8406.

47. Sukhishvili, S. A.; Kharlampieva, E.; Izumrudov, V., Where Polyelectrolyte Multilayers and Polyelectrolyte Complexes Meet. Macromolecules 2006, 39 (26), 88738881. 
48. Li, Y.-C.; Schulz, J.; Mannen, S.; Delhom, C.; Condon, B.; Chang, S.; Zammarano, M.; Grunlan, J. C., Flame Retardant Behavior of Polyelectrolyte-Clay Thin Film Assemblies on Cotton Fabric. ACS Nano 2010, 4 (6), 3325-3337.

49. Izumrudov, V. A.; Mussabayeva, B. K.; Murzagulova, K. B., Polyelectrolyte multilayers: preparation and applications. Russian Chemical Reviews 2018, 87 (2), 192200.

50. Khan, S. A.; Khan, S. B.; Khan, L. U.; Farooq, A.; Akhtar, K.; Asiri, A. M., Fourier Transform Infrared Spectroscopy: Fundamentals and Application in Functional Groups and Nanomaterials Characterization. In Handbook of Materials Characterization, Sharma, S. K., Ed. Springer International Publishing: Cham, 2018; pp 317-344.

51. Saliev, T.; Begimbetova, D.; Masoud, A.-R.; Matkarimov, B., Biological effects of non-ionizing electromagnetic fields: Two sides of a coin. Progress in Biophysics and Molecular Biology 2019, 141, 25-36.

52. Beasley, M. M.; Bartelink, E. J.; Taylor, L.; Miller, R. M., Comparison of transmission FTIR, ATR, and DRIFT spectra: implications for assessment of bone bioapatite diagenesis. Journal of Archaeological Science 2014, 46, 16-22.

53. Moraes, L. G. P.; Rocha, R. S. F.; Menegazzo, L. M.; de Araújo, E. B.; Yukimito, K.; Moraes, J. C. S., Infrared spectroscopy: a tool for determination of the degree of conversion in dental composites. J Appl Oral Sci 2008, 16 (2), 145-149.

54. Ausili, A.; Sánchez, M.; Gómez-Fernández, J., Attenuated total reflectance infrared spectroscopy: A powerful method for the simultaneous study of structure and 
spatial orientation of lipids and membrane proteins. Biomedical Spectroscopy and Imaging 2015, 4, 159-70.

55. Ricci, m. A.; Bruni, F.; Gallo, P.; Rovere, M.; K Soper, A., Water in confined geometries: Experiments and simulations. 2000; Vol. 12.

56. Wong, J. E.; Rehfeldt, F.; Hänni, P.; Tanaka, M.; Klitzing, R. v., Swelling Behavior of Polyelectrolyte Multilayers in Saturated Water Vapor. Macromolecules 2004, 37 (19), 7285-7289.

57. Kolasińska, M.; Krastev, R.; Gutberlet, T.; Warszyński, P. In Swelling and Water Uptake of PAH/PSS Polyelectrolyte Multilayers, Berlin, Heidelberg, Springer Berlin Heidelberg: Berlin, Heidelberg, 2008; pp 30-38.

58. Zhang, R.; Zhang, Y.; Antila, H. S.; Lutkenhaus, J. L.; Sammalkorpi, M., Role of Salt and Water in the Plasticization of PDAC/PSS Polyelectrolyte Assemblies. The Journal of Physical Chemistry B 2017, 121 (1), 322-333.

59. Michaels, A. S., POLYELECTROLYTE COMPLEXES. Industrial \& Engineering Chemistry 1965, 57 (10), 32-40.

60. Huglin, M. B.; Webster, L.; Robb, I. D., Complex formation between poly(4vinylpyridinium chloride) and poly[sodium(2-acrylamido-2-methyl propane sulfonate)] in dilute aqueous solution. Polymer 1996, 37 (7), 1211-1215.

61. Schlenoff, J. B.; Rmaile, A. H.; Bucur, C. B., Hydration Contributions to Association in Polyelectrolyte Multilayers and Complexes: Visualizing Hydrophobicity. Journal of the American Chemical Society 2008, 130 (41), 13589-13597. 
62. Sukhishvili, S. A.; Granick, S., Layered, Erasable Polymer Multilayers Formed by Hydrogen-Bonded Sequential Self-Assembly. Macromolecules 2002, 35 (1), 301-310.

63. Wang, Y.; Shen, Y.; Zhang, Y.; Yue, B.; Wu, C., pH-Sensitive Polyacrylic Acid (PAA) Hydrogels Trapped with Polysodium-p-Styrenesulfonate (PSS). Journal of Macromolecular Science, Part B 2006, 45, 563-571.

64. Dubas, S. T.; Schlenoff, J. B., Swelling and Smoothing of Polyelectrolyte Multilayers by Salt. Langmuir 2001, 17 (25), 7725-7727.

65. Abbott, S. B.; de Vos, W. M.; Mears, L. L. E.; Barker, R.; Richardson, R. M.; Prescott, S. W., Hydration of Odd-Even Terminated Polyelectrolyte Multilayers under Mechanical Confinement. Macromolecules 2014, 47 (10), 3263-3273.

66. Eneh, C. I.; Bolen, M. J.; Suarez-Martinez, P. C.; Bachmann, A. L.; Zimudzi, T. J.; Hickner, M. A.; Batys, P.; Sammalkorpi, M.; Lutkenhaus, J. L., Fourier transform infrared spectroscopy investigation of water microenvironments in polyelectrolyte multilayers at varying temperatures. Soft Matter 2020, 16 (9), 2291-2300.

67. Reid, D. K.; Summers, A.; O’Neal, J.; Kavarthapu, A. V.; Lutkenhaus, J. L., Swelling and Thermal Transitions of Polyelectrolyte Multilayers in the Presence of Divalent Ions. Macromolecules 2016, 49 (16), 5921-5930.

68. Gao, C.; Leporatti, S.; Moya, S.; Donath, E.; Moehwald, H., Swelling and Shrinking of Polyelectrolyte Microcapsules in Response to Changes in Temperature and Ionic Strength. Chemistry (Weinheim an der Bergstrasse, Germany) 2003, 9, 915-20.

69. Schwarz, B.; Schönhoff, M., Surface Potential Driven Swelling of Polyelectrolyte Multilayers. Langmuir 2002, 18 (8), 2964-2966. 
70. Salomäki, M.; Kankare, J., Specific Anion Effect in Swelling of Polyelectrolyte Multilayers. Macromolecules 2008, 41 (12), 4423-4428.

71. Köhler, K.; Biesheuvel, M.; Weinkamer, R.; Moehwald, H.; Sukhorukov, G., Salt-Induced Swelling-to-Shrinking Transition in Polyelectrolyte Multilayer Capsules. Physical review letters 2006, 97, 188301.

72. McCormick, M.; Smith, R. N.; Graf, R.; Barrett, C. J.; Reven, L.; Spiess, H. W., NMR Studies of the Effect of Adsorbed Water on Polyelectrolyte Multilayer Films in the Solid State. Macromolecules 2003, 36 (10), 3616-3625.

73. Parveen, N.; Schönhoff, M., Quantifying and controlling the cation uptake upon hydrated ionic liquid-induced swelling of polyelectrolyte multilayers. Soft Matter 2017, 13 (10), 1988-1997.

74. Koehler, R.; Steitz, R.; von Klitzing, R., About different types of water in swollen polyelectrolyte multilayers. Advances in Colloid and Interface Science 2014, 207, 325331.

75. Batys, P.; Zhang, Y.; Lutkenhaus, J. L.; Sammalkorpi, M., Hydration and Temperature Response of Water Mobility in Poly(diallyldimethylammonium)Poly(sodium 4-styrenesulfonate) Complexes. Macromolecules 2018, 51 (20), 8268-8277. 76. Zerball, M.; Laschewsky, A.; von Klitzing, R., Swelling of Polyelectrolyte Multilayers: The Relation Between, Surface and Bulk Characteristics. The Journal of Physical Chemistry B 2015, 119 (35), 11879-11886. 
77. Steitz, R.; Leiner, V.; Siebrecht, R.; v. Klitzing, R., Influence of the ionic strength on the structure of polyelectrolyte films at the solid/liquid interface. Colloids and Surfaces A: Physicochemical and Engineering Aspects 2000, 163 (1), 63-70.

78. Dodoo, S.; Steitz, R.; Laschewsky, A.; von Klitzing, R., Effect of ionic strength and type of ions on the structure of water swollen polyelectrolyte multilayers. Physical Chemistry Chemical Physics 2011, 13 (21), 10318-10325.

79. Mitra, S. K.; Dass, N.; Varshneya, N. C., Temperature Dependence of the Refractive Index of Water. The Journal of Chemical Physics 1972, 57 (4), 1798-1799.

80. Steitz, R.; Leiner, V.; Tauer, K.; Khrenov, V.; v. Klitzing, R., Temperatureinduced changes in polyelectrolyte films at the solid-liquid interface. Applied Physics A 2002, 74 (1), s519-s521.

81. Köhler, K.; Möhwald, H.; Sukhorukov, G. B., Thermal Behavior of Polyelectrolyte Multilayer Microcapsules: 2. Insight into Molecular Mechanisms for the PDADMAC/PSS System. The Journal of Physical Chemistry B 2006, 110 (47), 2400224010.

82. Nestler, P.; Block, S.; Helm, C. A., Temperature-Induced Transition from OddEven to Even-Odd Effect in Polyelectrolyte Multilayers Due to Interpolyelectrolyte Interactions. The Journal of Physical Chemistry B 2012, 116 (4), 1234-1243.

83. Lutkenhaus, J. L.; Hrabak, K. D.; McEnnis, K.; Hammond, P. T., Elastomeric Flexible Free-Standing Hydrogen-Bonded Nanoscale Assemblies. Journal of the American Chemical Society 2005, 127 (49), 17228-17234. 
84. Smedley, S. B.; Zimudzi, T. J.; Chang, Y.; Bae, C.; Hickner, M. A., Spectroscopic Characterization of Sulfonate Charge Density in Ion-Containing Polymers. The Journal of Physical Chemistry B 2017, 121 (51), 11504-11510.

85. Kitadai, N.; Sawai, T.; Tonoue, R.; Nakashima, S.; Katsura, M.; Fukushi, K., Effects of Ions on the OH Stretching Band of Water as Revealed by ATR-IR Spectroscopy. Journal of Solution Chemistry 2014, 43 (6), 1055-1077.

86. Moilanen, D. E.; Piletic, I. R.; Fayer, M. D., Tracking Water's Response to Structural Changes in Nafion Membranes. The Journal of Physical Chemistry A 2006, 110 (29), 9084-9088.

87. Park, S.; Fayer, M. D., Hydrogen bond dynamics in aqueous NaBr solutions. Proc Natl Acad Sci U S A 2007, 104 (43), 16731-16738.

88. Smedley, S. B.; Chang, Y.; Bae, C.; Hickner, M. A., Measuring water hydrogen bonding distributions in proton exchange membranes using linear Fourier Transform Infrared spectroscopy. Solid State Ionics 2015, 275, 66-70.

89. Brubach, J.-B.; Mermet, A.; Filabozzi, A.; Gerschel, A.; Roy, P., Signatures of the hydrogen bonding in the infrared bands of water. The Journal of Chemical Physics 2005, 122 (18), 184509.

90. Schmidt, J. R.; Corcelli, S. A.; Skinner, J. L., Pronounced non-Condon effects in the ultrafast infrared spectroscopy of water. The Journal of Chemical Physics 2005, 123 (4), 044513.

91. Yang, J. C.; Jablonsky, M. J.; Mays, J. W., NMR and FT-IR studies of sulfonated styrene-based homopolymers and copolymers. Polymer 2002, 43 (19), 5125-5132. 
92. Wang, Y.; Shen, Y.; Zhang, Y.; Yue, B.; Wu, C., pH-Sensitive Polyacrylic Acid (PAA) Hydrogels Trapped with Polysodium-p-Styrenesulfonate (PSS). 2006; Vol. 45, $\mathrm{p}$ 563-571.

93. Falk, M.; Ford, T. A., INFRARED SPECTRUM AND STRUCTURE OF LIQUID WATER. Canadian Journal of Chemistry 1966, 44 (14), 1699-1707.

94. Batys, P.; Kivistö, S.; Lalwani, S. M.; Lutkenhaus, J. L.; Sammalkorpi, M., Comparing water-mediated hydrogen-bonding in different polyelectrolyte complexes. Soft Matter 2019, 15 (39), 7823-7831.

95. Wang, F.; Yang, J.; Zhao, J., Understanding anti-polyelectrolyte behavior of a well-defined polyzwitterion at the single-chain level. Polymer International 2015, 64 (8), 999-1005.

96. Antila, H. S.; Sammalkorpi, M., Polyelectrolyte Decomplexation via Addition of Salt: Charge Correlation Driven Zipper. The Journal of Physical Chemistry B 2014, 118 (11), 3226-3234.

97. Antila, H. S.; Härkönen, M.; Sammalkorpi, M., Chemistry specificity of DNApolycation complex salt response: a simulation study of DNA, polylysine and polyethyleneimine. Physical Chemistry Chemical Physics 2015, 17 (7), 5279-5289.

98. Ninno, A.; Congiu Castellano, A.; Del Giudice, E., The supramolecular structure of liquid water and quantum coherent processes in biology. 2013; Vol. 442, p 012031.

99. Ninno, A.; Del Giudice, E.; Gamberale, L.; Congiu Castellano, A., The Structure of Liquid Water Emerging from the Vibrational Spectroscopy: Interpretation with QED Theory. 2013. 
100. Walrafen, G. E.; Fisher, M. R.; Hokmabadi, M. S.; Yang, W. H., Temperature dependence of the low- and high-frequency Raman scattering from liquid water. The Journal of Chemical Physics 1986, 85 (12), 6970-6982.

101. Walrafen, G. E., Raman Spectral Studies of the Effects of Electrolytes on Water. The Journal of Chemical Physics 1962, 36 (4), 1035-1042.

102. Carey, D. M.; Korenowski, G. M., Measurement of the Raman spectrum of liquid water. The Journal of Chemical Physics 1998, 108 (7), 2669-2675.

103. Krebs, T.; Tan, H. L.; Andersson, G.; Morgner, H.; Gregory Van Patten, P., Increased layer interdiffusion in polyelectrolyte films upon annealing in water and aqueous salt solutions. Physical chemistry chemical physics : PCCP 2006, 8 (46), 54628. 